\title{
Gevrey regularizing effect for the (generalized) Korteweg-de Vries equation and nonlinear Schrödinger equations
}

by

\author{
Anne DE BOUARD \\ C.N.R.S. et Université de Paris-Sud, Mathématique, \\ Bâtiment 425, 91405 Orsay, France. \\ Nakao HAYASHI \\ Department of Mathematics, Faculty of Engineering, \\ Gunma University, Kiryu 376, Japan. \\ and

\section{Keiichi KATO} \\ Department of Mathematics, Faculty of Science, \\ Osaka University, Toyonaka 560, Japan.
}

ABSTRACT. - This paper is concerned with regularizing effects of solutions to the (generalized) Korteweg-de Vries equation

$(\mathrm{gKdV})$

$$
\left\{\begin{array}{l}
\partial_{t} u+\partial_{x}^{3} u=\lambda u^{p-1} \partial_{x} u, \quad(t, x) \in \mathbb{R} \times \mathbb{R} \\
u(0)=\phi, \quad x \in \mathbb{R}
\end{array}\right.
$$

and nonlinear Schrödinger equations in one space dimension

(NLS)

$$
\left\{\begin{array}{l}
i \partial_{t} u+\frac{1}{2} \partial_{x}^{2} u=G(u, \bar{u}), \quad(t, x) \in \mathbb{R} \times \mathbb{R}, \\
u(0)=\psi, \quad x \in \mathbb{R},
\end{array}\right.
$$

where $p$ is an integer satisfying $p \geq 2, \lambda \in \mathbb{C}$ and $G$ is a polynomial of $(u, \bar{u})$. We prove that if the initial function $\phi$ is in a Gevrey class of order 3 defined in Section 1, then there exists a positive time $T$ such that the solution of $(\mathrm{gKdV})$ is analytic in space variable for $t \in[-T, T] \backslash\{0\}$, and if the initial function $\psi$ in a Gevrey class of order 2 , then there exists a 
positive time $T$ such that the solution of (NLS) is analytic in space variable for $t \in[-T, T] \backslash\{0\}$.

RESUMÉ. - Nous étudions, dans cet article, certains effets régularisants pour les solutions de l'équation de Korteweg-de Vries (généralisée)

$(\mathrm{gKdV})$

$$
\left\{\begin{array}{l}
\partial_{t} u+\partial_{x}^{3} u=\lambda u^{p-1} \partial_{x} u, \quad(t, x) \in \mathbb{R} \times \mathbb{R}, \\
u(0)=\phi, \quad x \in \mathbb{R},
\end{array}\right.
$$

et des équations de Schrödinger monodimensionnelles

$$
\left\{\begin{array}{l}
i \partial_{t} u+\frac{1}{2} \partial_{x}^{2} u=G(u, \bar{u}), \quad(t, x) \in \mathbb{R} \times \mathbb{R}, \\
u(0)=\psi, \quad x \in \mathbb{R}
\end{array}\right.
$$

où $p$ est un entier supérieur ou égal à $2, \lambda \in \mathbb{C}$ et $G$ est un polynôme en $(u, \bar{u})$. Nous montrons que, lorsque la donnée initiale $\phi$ appartient à la classe de Gevrey définie dans la première partie, il existe un temps $T$ tel que la solution de (gKdV) est analytique en espace pour $t \in[-T, T] \backslash\{0\}$; de même, lorsque la donnée initiale $\psi$ appartient à une certaine classe de Gevrey d'ordre 2, il existe un temps $T$ tel que la solution de (NLS) est analytique en espace pour $t \in[-T, T] \backslash\{0\}$.

\section{INTRODUCTION}

In this paper we study regularizing effects of solutions to the (generalized) Korteweg-de Vries equation

$$
\left\{\begin{array}{l}
\partial_{t} u+\partial_{x}^{3} u=\lambda u^{p-1} \partial_{x} u, \quad(t, x) \in \mathbb{R} \times \mathbb{R}, \\
u(0)=\phi, \quad x \in \mathbb{R},
\end{array}\right.
$$

and nonlinear Schrödinger equations in one space dimension

$$
\left\{\begin{array}{l}
i \partial_{t} u+\frac{1}{2} \partial_{x}^{2} u=G(u, \bar{u}), \quad(t, x) \in \mathbb{R} \times \mathbb{R}, \\
u(0)=\psi, \quad x \in \mathbb{R},
\end{array}\right.
$$

where $p$ is an integer satisfying $p \geq 2, \lambda \in \mathbb{C}$ and $G$ is a polynomial of $(u, \bar{u})$. We prove that if the initial function $\phi$ is in a Gevrey class of order 3 defined below, then there exists a positive time $T$ such that the solution of $(\mathrm{gKdV})$ is analytic in space variable for $t \in[-T, T] \backslash\{0\}$, and if the initial function $\psi$ is in a Gevrey class of order 2 , then there exists a positive 
time $T$ such that the solution of (NLS) is analytic in space variable for $t \in[-T, T] \backslash\{0\}$. In other words, the singularities of the data go to infinity at once. We call this property the Gevrey regularizing effect.

We also prove analyticity in time of solutions of $(\mathrm{gKdV})$ which is the same result as that in [H-K.K] in the case of (NLS).

To state our results precisely we introduce the basic function space used in this paper. We define a Gevrey class of order $\sigma$ as follows :

$$
\begin{aligned}
& G_{\sigma}^{A_{1} A_{2} \cdots A_{N}}\left(P_{1}, P_{2}, \cdots, P_{N} ; X\right) \\
& =\left\{f \in X ;\|f\|_{G_{\sigma}^{A_{1} A_{2} \cdots A_{N}}\left(P_{1}, P_{2}, \cdots, P_{N} ; X\right)}\right. \\
& \left.\quad=\sum_{j_{1}, \cdots, j_{N}=0}^{\infty} \frac{A_{1}^{j_{1}}}{\left(j_{1} !\right)^{\sigma}} \cdots \frac{A_{N}^{j_{N}}}{\left(j_{N} !\right)^{\sigma}}\left\|P_{1}^{j_{1}} P_{2}^{j_{2}} \cdots P_{N}^{j_{N}} f\right\|_{X}<\infty\right\},
\end{aligned}
$$

where $A_{1}, \cdots, A_{N}$ are positive constants, $P_{1}, \cdots, P_{N}$ are vector fields with analytic coefficients and $X$ is a Banach space of functions on an open set in $\mathbb{R}^{n}$ with norm $\|\cdot\|_{X}$ and $\sigma \geq 1$. The above function space is used to define several function spaces :

$$
Y_{T}=\left\{f \in C\left([-T, T] ; L^{2}\right) ;\|f \mid\|_{Y_{T}}<\infty\right\},
$$

where

\|\|$f \|_{Y_{T}}=\max \left\{\gamma(T, P, 3, f), \sigma(T, P, 3, f), \gamma\left(T, P, 0, \partial_{t} f\right), \sigma\left(T, P, 0, \partial_{t} f\right)\right\}$

with

$$
\begin{gathered}
P=x \partial_{x}+3 t \partial_{t} \\
\gamma(T, P, m, f)=\sum_{k, l=0}^{\infty} \frac{A_{1}^{l}}{(l !)^{\sigma}} \frac{A_{2}^{k}}{(k !)^{\sigma}} \sup _{t \in[-T, T]}\left\|P^{l} \partial_{x}^{k} f(t)\right\|_{H^{m}}
\end{gathered}
$$

and

$$
\begin{gathered}
\sigma(T, P, m, f)=\sum_{j=0}^{m} \sum_{k, l=0}^{\infty} \frac{A_{1}^{l}}{(l !)^{\sigma}} \frac{A_{2}^{k}}{(k !)^{\sigma}} \sup _{x}\left(\int_{-T}^{T}\left|\partial_{x}^{j+1} P^{l} \partial_{x}^{k} f\right|^{2} d t\right)^{1 / 2}, \\
Z_{\infty}=\left\{f \in C\left(\mathbb{R} ; L^{2}\right) ;\|f\| \|_{Z_{\infty}}<\infty\right\},
\end{gathered}
$$

where

$$
\|\| f \|_{z_{\infty}}=\max \left\{\left|\|f \mid\|_{Y_{\infty}}, \mu(f)\right\}\right.
$$


with

$$
\begin{gathered}
\mu(f)=\sum_{l, k=0}^{\infty} \frac{A_{1}^{l}}{(l !)^{\sigma}} \frac{A_{2}^{k}}{(k !)^{\sigma}} \sup _{t \in \mathbb{R}}(1+|t|)^{1 / 3}\left\|P^{l} \partial_{x}^{k} f(t)\right\|_{H^{1, \infty}} . \\
H^{m, p}(-R, R)=\left\{f \in L^{p}(-R, R) ;\|f\|_{H^{m, p}(-R, R)}\right. \\
\left.=\sum_{0 \leq j \leq m}\left\|\partial_{x}^{j} f\right\|_{L^{p}(-R, R)}<\infty\right\} .
\end{gathered}
$$

For simplicity we let $H^{m}(-R, R)=H^{m, 2}(-R, R), H^{m, p}=H^{m, p}(\mathbb{R})$ and $H^{m}=H^{m, 2}$. We also define the closed balls in $Y_{T}$ and $Z_{\infty}$ as follows.

$$
Y_{T, \rho}=\left\{f \in Y_{T} ;\|f\| \|_{Y_{T}} \leq \rho\right\}, Z_{\infty, \rho}=\left\{f \in Z_{\infty} ;\|f\| \|_{Z_{\infty}} \leq \rho\right\}
$$

Throughout this paper we assume that $A_{1}<1$ since when $\sigma=1$ Aikawa's result [A,Theorem 3] says that If $A_{1}>1$, then $G_{1}^{A_{1}}\left(x \partial_{x} ; L^{2}\right)=$ $\{0\}$ which implies that the solutions constructed in the theorems below are identically zero when $A_{1}>1$ and $\sigma=1$. Different positive constants are denoted by $C, C_{0}, C_{1}, \cdots, A_{1}, A_{2}, \cdots$ in Sections 1 and 4 .

We now state our main results.

THEOREM 1.1. (gKdV). - We assume that $\sigma \geq 1$ and

$$
\phi \in G_{\sigma}^{A_{1} A_{2}}\left(x \partial_{x}, \partial_{x} ; H^{3}\right) .
$$

Then there exist positive constants $A_{3}, A_{4}, T$ and a unique solution $u$ of ( $g K d V)$ such that

$$
u \in C\left([-T, T] ; H^{3}\right)
$$

and

$$
\begin{aligned}
& u \in G_{\sigma}^{|t| A_{3}}\left(\partial_{t} ; H^{3}(-R, R)\right) \cap G_{\max (\sigma / 3,1)}^{|t|^{1 / 3} A_{4}}\left(\partial_{x} ; H^{3}(-R, R)\right) \\
& \text { for } t \in[-T, T]
\end{aligned}
$$

where

$$
A_{3}<\min \left\{\frac{3 A_{1}}{1+3 A_{1}}, \frac{3 A_{2}}{4 A_{2}+e^{1 / 3}(1+R)}\right\},
$$

$A_{4}$ depends on $A_{3}$ and

$$
\|u\|_{G_{\sigma}^{|t| A_{3}}\left(\partial_{t} ; H^{3}(-R, R)\right)} .
$$


THEOREM 1.1'. (gKdV). - We assume that $\sigma \geq 1$ and

$$
\phi \in G_{\sigma}^{A_{1} A_{2}}\left(x \partial_{x}, \partial_{x} ; H^{3}\right) \cap G_{\sigma}^{A_{1} A_{2}}\left(x \partial_{x}, \partial_{x} ; H^{1,1}\right) .
$$

Furthermore we assume that

$$
\epsilon_{1}=\|\phi\|_{G_{\sigma}^{A_{1} A_{2}}\left(x \partial_{x}, \partial_{x} ; H^{3}\right)}+\|\phi\|_{G_{\sigma}^{A_{1} A_{2}}\left(x \partial_{x}, \partial_{x} ; H^{1,1}\right)}
$$

is sufficiently small and $p$ is an integer satisfying $p \geq 6$. Then there exist positive constants $A_{3}, A_{4}$ and a unique solution $u$ of $(\mathrm{gKdV})$ such that

$$
u \in C\left(\mathbb{R} ; H^{3}\right)
$$

$u \in G_{\sigma}^{|t| A_{3}}\left(\partial_{t} ; H^{3}(-R, R)\right) \cap G_{\max (\sigma / 3,1)}^{|t|^{1 / 3} A_{4}}\left(\partial_{x} ; H^{3}(-R, R)\right) \quad$ for any $\quad t \in \mathbb{R}$, $\|u(t)\|_{G_{\sigma}^{|t| A_{3}}\left(\partial_{t} ; H^{1, \infty}(-R, R)\right)} \leq$ const. $\epsilon_{1}(1+|t|)^{-1 / 3} \quad$ for any $\quad t \in \mathbb{R}$,

where

$$
A_{3}<\min \left\{\frac{3 A_{1}}{1+3 A_{1}}, \frac{3 A_{2}}{4 A_{2}+e^{1 / 3}(1+R)}\right\},
$$

$A_{4}$ depends on $A_{3}$ and

$$
\|u\|_{G_{o}^{|t| A_{3}}\left(\partial_{t} ; H^{3}(-R, R)\right)} .
$$

Remark 1.1. - The positive constants appearing in Theorems 1.1 and 1.1' are important numbers which determine the domain on which the data and the solution of $(\mathrm{gKdV})$ have analytic continuations when $\sigma=1$ or 3 . We explain this point herein.

(I) $([\mathrm{H}-\mathrm{K} . \mathrm{K}])$. If $\phi$ has an analytic continuation $\Phi$ on the complex domain

$$
\begin{aligned}
& \Gamma_{\sqrt{2} A_{1}, A_{2}}=\{z \in \mathbb{C} ; z=x+i y,-\infty<x<\infty \\
&\left.-A_{2}-(\tan \alpha)|x|<y<A_{2}+(\tan \alpha)|x|, A_{2}>0\right\},
\end{aligned}
$$

where $0<\alpha=\sin ^{-1} \sqrt{2} A_{1}<\pi / 2$ and

$$
\int_{\Gamma_{\sqrt{2} A_{1} A_{2}}}|\Phi(z)|^{2} d x d y<\infty
$$

then

$$
\phi \in G_{1}^{A_{1} A_{2}}\left(x \partial_{x}, \partial_{x} ; H^{3}\right)
$$


(II) In the case $\sigma=1$, Theorems $1.1-1.1$ ' say that

$$
u \in G_{1}^{|t| A_{3}}\left(\partial_{t} ; H^{3}(-R, R)\right)
$$

which implies

$$
\sum_{l=0}^{\infty} \frac{\left(|t| A_{3}\right)^{l}}{l !}\left|\partial_{t}^{l} u(t, x)\right|<\infty \quad \text { for } \quad x \in(-R, R) .
$$

Hence $u$ has an analytic continuation $U\left(z_{0}, x\right)$ on the complex plane

$$
\left\{z_{0} \in \mathbb{C} ; z_{0}=t+i \tau ;\left|\arg z_{0}\right|<\sin ^{-1} A_{3}\right\} \quad \text { for } \quad x \in(-R, R) \text {. }
$$

From Theorems 1.1-1.1' we see that $A_{3}$ is bounded from above by an upper limit $3 / 4\left(A_{1}=1, A_{2}=\infty\right)$. On the other hand in the case of the nonlinear heat equation

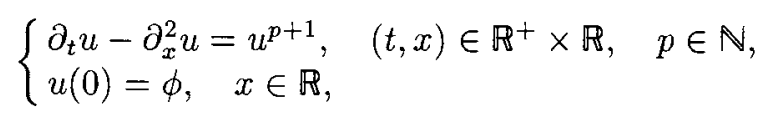

it is well known that if $\phi \in H^{1}(\mathbb{R})$, then $u$ has an analytic continuation $U\left(z_{0}, x\right)$ on the complex domain

$$
\left\{z_{0} \in \mathbb{C} ; z_{0}=t+i \tau ;\left|\arg z_{0}\right|<\pi / 2\right\} \quad \text { for } \quad x \in \mathbb{R} \text {. }
$$

This last result corresponds to the case $A_{3}=1$. Hence the following question arises. Can we improve the upper limit on $A_{3}$ ?

(III) In the case $\sigma=3$, Theorems 1.1-1.1' say that if the initial function $\phi$ belongs to a Gevrey class of order 3 , the solution $u(t, x)$ of $(\mathrm{gKdV})$ has an analytic continuation $U(t, z)$ on the complex domain

$$
\left\{z \in \mathbb{C} ; z=x+i y,|x|<R,-|t|^{1 / 3} A_{4}<y<|t|^{1 / 3} A_{4}, t \neq 0\right\} .
$$

In the case of the KdV equation ( $p=2$ ), the end of the proof of Theorem 1.1 (Section 4 below) shows that it is sufficient to take $A_{4}$ such that

$$
A_{4}<3\left(\frac{A_{3}}{2\left(1+\left(1+2^{4} \cdot 3^{18}|\lambda|^{2} C^{2} C_{0}^{2} C_{1}\right) A_{3}\right)}\right)^{1 / 3}
$$

where

$$
C_{0}=\|u\|_{G_{\sigma}^{|t| A_{3}}\left(\partial_{t} ; H^{3}(-R, R)\right\}}, \quad C_{1}=1+\sum_{m=1}^{\infty} m^{-4 / 3}
$$


and $C$ is the best constant arising in the Sobolev's inequality

$$
\|\cdot\|_{L^{\infty}(-R, R)} \leq \frac{C}{4}\|\cdot\|_{H^{1}(-R, R)} .
$$

(The constant $C$ can be given explicitly since we have

$$
\left.\|\cdot\|_{L^{\infty}(-R, R)} \leq\left(2 R+\frac{1}{2 R}\right)^{1 / 2}\|\cdot\|_{H^{1}(-R, R)} .\right)
$$

We now give a typical example of the function in $G_{3}^{A_{1} A_{2}}\left(x \partial_{x}, \partial_{x} ; H^{3}\right)$, but is not an analytic function, which may help the readers to understand the Gevrey regularizing effect.

We put

$$
\varphi(x)=\left\{\begin{array}{l}
\exp \left(-x^{-\frac{1}{s-1}}\right), \quad x>0, \\
0, \quad x \leq 0
\end{array}\right.
$$

Then the function $\varphi(x)$ belongs to Gevrey class of order $s$ but not belong to Gevrey class of order $r$, where $1 \leq r<s$ (see [Ko, p41, Lemma 2.1] for the proof). We let $\psi(x)=\varphi\left(1-x^{2}\right)$ and $s=3$. Then there exist positive constants $A_{1}$ and $A_{2}$ such that $\psi(x) \in G_{3}^{A_{1} A_{2}}\left(x \partial_{x}, \partial_{x} ; H^{3}\right)$, but $\psi(x)$ is not analytic in the neighborhoods of \pm 1 . More precisely $\psi(x)$ is not in $G_{r}^{A_{1} A_{2}}\left(x \partial_{x}, \partial_{x} ; H^{3}\right)$ for $1 \leq r<3$ and any $A_{1}, A_{2}>0$.

The analyticity of solutions of (gKdV) was studied first by $\mathrm{T}$. Kato and K. Masuda [Ka-M] (see also $[\mathrm{H}]$ ). They proved that if the initial function $\phi$ is in $G_{1}^{A_{1}}\left(\partial_{x} ; H^{3}\right)$, then there exist $A_{1}^{\prime}>0, T>0$ and a unique solution $u$ of $(\mathrm{gKdV})$ such that

$$
u \in C\left([-T, T] ; H^{3}\right)
$$

and

$$
u \in G_{1}^{A_{1}^{\prime}}\left(\partial_{x} ; H^{3}\right) \text { for } t \in[-T, T] \text { and } A_{1}^{\prime}<A_{1} .
$$

Their method requires the condition $\sigma=1$ to treat the nonlinear term involving the space derivative of the solution $u$. Hence their method is not applicable to the general case $\sigma \geq 1$. To overcome this difficulty we use the local smoothing property of solutions to the Airy equation $\partial_{t} u+\partial_{x}^{3} u=0$ which was shown by [Ke-P-V 1] first. Local smoothing property enables us to handle $(\mathrm{gKdV})$ by using the contraction mapping principle (see [Ke$\mathrm{P}-\mathrm{V}$ 5]). In fact, in the same way as in the proof of Proposition 3.1 in 
Section 3 we can prove that there exist $T>0$ and a unique solution $u$ of $(\mathrm{gKdV})$ such that

$$
u \in C\left([-T, T] ; H^{3}\right)
$$

and

$$
u \in G_{\sigma}^{A_{1}}\left(\partial_{x} ; H^{3}\right) \text { for } t \in[-T, T],
$$

when $\phi \in G_{\sigma}^{A_{1}}\left(\partial_{x} ; H^{3}\right)(\sigma \geq 1)$. From the above result we can prove that there exists $A_{2}>0$ such that

$$
u \in G_{3 \sigma}^{A_{2}}\left(\partial_{t} ; H^{3}\right) \text { for } t \in[-T, T] .
$$

However analyticity in time of solutions to ( $\mathrm{gKdV})$ does not come from (1.2) since $\sigma \geq 1$ is needed to obtain (1.1).

In [Ka], it was shown that if $\phi \in L_{b}^{2} \equiv H^{2} \cap L^{2}\left(e^{b x} d x\right)(b>0)$, then the solution of $(\mathrm{gKdV})$ becomes $C^{\infty}(-R, R)$ for $t>0$ in space variable. His proof is based on the fact that the unitary group $\exp \left(-t \partial_{x}^{3}\right)$ in $L_{b}^{2}$ is equivalent to

$$
\exp \left(-t\left(\partial_{x}-b\right)^{3}\right)=\exp \left(-t \partial_{x}^{3}\right) \exp \left(-3 b t \partial_{x}^{2}\right) \exp \left(-3 b^{2} t \partial_{x}\right) \exp \left(-b^{3} t\right)
$$

in $L^{2}$ when $t>0$. Hence the method is not valid for the negative time and it is not clear whether or not the solution of $(\mathrm{KdV})$ becomes analytic for $t>0$.

In [Cr-Kap-St], the authors studied a fully nonlinear equation of $\mathrm{KdV}$ type in one space dimension :

$$
\partial_{t} u+f\left(\partial_{x}^{3} u, \partial_{x}^{2} u, \partial_{x} u, u, x, t\right)=0, \quad x \in \mathbb{R},
$$

where $f \in C^{\infty}$ and

$$
\partial f / \partial\left(\partial_{x}^{3} u\right) \geq C>0 \quad \text { and } \quad \partial f / \partial\left(\partial_{x}^{2} u\right) \leq 0 .
$$

They showed that if the initial function decays faster than any polynomial on $\mathbb{R}^{+}$, and possesses certain minimal regularity, then the solution $u(t, \cdot) \in C^{\infty}(-R, R)$ for $t>0$. Their result ([Cr-Kap-St, Theorem 2.1]) is considered as a generalization of results of $[\mathrm{Ka}]$ and $[\mathrm{Kr}-\mathrm{F}]$.

We prove the regularity in time result for solutions to (gKdV) implying analyticity by using the operator $P=x \partial_{x}+3 t \partial_{t}$ (which almost commutes with the operator $\partial_{t}+\partial_{x}^{3}$ ) and the local smoothing property. We also prove a global existence in time result for solutions in a Gevrey class implying analyticity by using a similar method to that of W. A. Strauss [St]. 
By making use of the regularity in time result obtained in Section 3 and an induction argument, we prove regularity in space of solutions to ( $\mathrm{gKdV})$ yielding analyticity in space variable.

We next state the results concerning (NLS).

THEOREM 1.2. (NLS). - We assume that $\sigma \geq 1$ and

$$
\psi \in G_{\sigma}^{A_{1} A_{2}}\left(x \partial_{x}, \partial_{x} ; H^{2}\right) .
$$

Then there exist positive constants $A_{3}, A_{4}, T$ and a unique solution $u$ of (NLS) such that

$$
\begin{aligned}
& \qquad u \in C\left([-T, T] ; H^{2}\right), \\
& u \in G_{\sigma}^{|t| A_{3}}\left(\partial_{t} ; H^{2}(-R, R)\right) \cap G_{\max (\sigma / 2,1)}^{|t|^{1 / 2} A_{4}}\left(\partial_{x} ; H^{2}(-R, R)\right) \\
& \text { for } t \in[-T, T],
\end{aligned}
$$

where

$$
A_{3}<\min \left\{\frac{2 A_{1}}{1+2 A_{1}}, \frac{2 A_{2}}{3 A_{2}+e^{1 / 2}(1+R)}\right\},
$$

$A_{4}$ depends on $A_{3}$ and

$$
\|u\|_{G_{\sigma}^{|t| A_{3}}\left(\partial_{t} ; H^{2}(-R, R)\right)} .
$$

THEOREM 1.2'. (NLS). - We assume that $\sigma \geq 1$ and

$$
\psi \in G_{\sigma}^{A_{1} A_{2}}\left(x \partial_{x}, \partial_{x} ; H^{2}\right) \cap G_{\sigma}^{A_{1} A_{2}}\left(x \partial_{x}, \partial_{x} ; L^{1}\right) .
$$

Furthermore we assume that

$$
\epsilon_{1}=\|\psi\|_{G_{\sigma}^{A_{1} A_{2}}\left(x \partial_{x}, \partial_{x} ; H^{2}\right)}+\|\psi\|_{G_{\sigma}^{A_{1} A_{2}}\left(x \partial_{x}, \partial_{x} ; L^{1}\right)}
$$

is sufficiently small and $G(u, \bar{u})$ satisfies the following growth condition

$$
|G(s, \bar{s})| \leq \text { const. }|s|^{p} \quad \text { for } \quad|s|<1,
$$

where $p$ is an integer satisfying $p \geq 5$. Then there exist $A_{3}, A_{4}$ and a unique solution $u$ such that

$$
\begin{aligned}
& u \in C\left(\mathbb{R} ; H^{2}\right), \\
& \quad u \in G_{\sigma}^{|t| A_{3}}\left(\partial_{t} ; H^{2}(-R, R)\right) \cap G_{\max (\sigma / 2,1)}^{|t|^{1 / 2} A_{4}}\left(\partial_{x} ; H^{2}(-R, R)\right) \\
& \text { for any } t \in \mathbb{R}, \\
& \|u(t)\|_{G_{\sigma}^{|t| A_{3}}\left(\partial_{t} ; L^{\infty}(-R, R)\right)} \leq \text { const. } \epsilon_{1}(1+|t|)^{-1 / 2} \text { for any } t \in \mathbb{R},
\end{aligned}
$$


where

$$
A_{3}<\min \left\{\frac{2 A_{1}}{1+2 A_{1}}, \frac{2 A_{2}}{3 A_{2}+e^{1 / 2}(1+R)}\right\} \text {, }
$$

$A_{4}$ depends on $A_{3}$ and

$$
\|u\|_{G_{\sigma}^{|t| A_{3}}\left(\partial_{t} ; H^{2}(-R, R)\right)^{*}}
$$

Remark 1.2.

(I) By the same argument as in Remark 1.1 (I), we see that the solution $u$ of (NLS) has an analytic continuation $U\left(z_{0}, x\right)$ on the complex plane

$$
\left\{z_{0} \in \mathbb{C} ; z_{0}=t+i \tau ;\left|\arg z_{0}\right|<\sin ^{-1} A_{3}\right\} \quad \text { for } \quad x \in(-R, R)
$$

and $A_{3}$ has the upper limit $2 / 3$. The same question as in Remark 1.1 (I) arises concerning this upper limit.

(II) In the case $\sigma=2$, Theorems 1.2-1.2' say that if the initial function $\psi$ is in a Gevrey class of order 2 , the solution $u(t, x)$ of (NLS) has an analytic continuation $U(t, z)$ on the complex domain

$$
\left\{z \in \mathbb{C} ; z=x+i y,|x|<R,-|t|^{1 / 2} A_{4}<y<|t|^{1 / 2} A_{4}, t \neq 0\right\} .
$$

(III) In the case $G(u, \bar{u})=u^{2}$, the end of the proof of Theorem 1.2 given in Section 5 shows that it is sufficient to take $A_{4}$ such that

$$
A_{4}<2\left(\frac{A_{3}}{2\left(1+2^{9} \cdot 3 C C_{0} A_{3}\right)}\right)^{1 / 2}
$$

where

$$
C_{0}=\|u\|_{G_{\sigma}^{|t| A_{3}}\left(\partial_{t} ; H^{2}(-R, R)\right)}
$$

and $C$ is the best constant arising in the Sobolev's inequality

$$
\|\cdot\|_{L^{\infty}(-R, R)} \leq \frac{C}{4}\|\cdot\|_{H^{1}(-R, R)} .
$$

The smoothing property of solutions to (NLS) implying analyticity in space variable was studied in [H-Sai] in the case of $G(u, \bar{u})=|u|^{2 k} u$, $k \in \mathbb{N}$. More precisely, the main result of [H-Sai] is as follows. 
If the initial function $\phi$ satisfies

$$
\left\|\left(\cosh A_{4} x\right) \phi\right\|_{H^{1}}<\infty,
$$

then the solution $u(t, x)$ of (NLS) has an analytic continuation $U(t, x)$ on the complex plane

$$
\left\{z \in \mathbb{C} ; z=x+i y,-|t| A_{4}<y<|t| A_{4}, t \neq 0\right\} .
$$

The result stated in Remark 1.2 (I) was already obtained in [H-K.K] but the result stated in Remark 1.2 (II) is new. It is interesting to compare the result of [H-Sai] given above and Remark 1.2 (II). Our result is new even in the case $G(u, \bar{u})=|u|^{2 k} u, k \in \mathbb{N}$ since we do not assume that the initial function decays at infinity.

For a general class of equations which includes a large number of models arising in the context of water waves, analytic solutions were obtained in [B]. We note here that the methods used in [H-Sai], [H-K.K] are not sufficient to treat the nonlinear Schrödinger equation with a nonlinear term involving the derivative of the unknown function :

$$
i \partial_{t} u+\frac{1}{2} \partial_{x}^{2} u=G\left(u, \partial_{x} u, \bar{u}, \partial_{x} \bar{u}\right),
$$

where $G$ is also a polynomial of $\left(u, \partial_{x} u, \bar{u}, \partial_{x} \vec{u}\right)$.

The gauge transformation techniques used in [H-O] are applicable to prove a time regularity result similar to Theorem 1.2 for (1.3) and for general space dimension the method of [Ke-P-V 2] based on [Ke-P-V 2, Theorem 2.3 (2.9)] is applicable for (1.3) with a smallness condition on the data. However these methods cause undesireble complexities, and so we do not go into the problem (1.3) in this paper. The difficulty to handle (1.3) arises from the fact that the smoothing property of solutions to the linear homogeneous Schrödinger equation is not sufficient compared with the Airy equation (see , [Ke-P-V 2, Theorem 2.1]). Local smoothing properties in the usual Sobolev spaces for the linear Schrödinger equation were studied by [Co-Sau], [Sj] and [V] simultaneously. A sharp version of the local smoothing property was obtained in [Ke-P-V 1, Section 4]. Moreover they proved a sharp inhomogeneous version of the local smoothing property in [Ke-P-V 2, Theorem 2.3] for Schrödinger equations which was used to study several higher order models arising in both physics and mathematics in [Ke-P-V 3,4].

G. Ponce [P, Theorem 3.2, Theorem 4.2] studied the regularity of solutions to nonlinear dispersive equations including (gKdV) and (NLS) 
as examples. Roughly speaking, his results are as follows. If $\phi \in H^{2 k}$ and $\left(x \partial_{x}\right)^{j} \phi \in L^{2}(j=1, \cdots, k)$, then the solution of $(\mathrm{gKdV})$ is in $H^{3 k}(-R, R)$ for any $R$ in some time interval. If $\psi \in H^{k}$ and $\left(x \partial_{x}\right)^{j} \psi \in L^{2}$ $(j=1, \cdots, k)$, then the solution of (NLS) is in $H^{2 k}(-R, R)$ for any $R$ in some time interval. His methods are based on the classical energy method and the facts that the linear operator $\partial_{t}+\partial_{x}^{3}$ commutes with the operator $x+3 t \partial_{x}^{2}$ and the linear operator $i \partial_{t}+\frac{1}{2} \partial_{x}^{2}$ commutes with the operator $x+i t \partial_{x}$. However our results do not follow from the methods in [P]. This commutation property was used in $[\mathrm{G}-\mathrm{Vel}]$ to study the scattering theory for nonlinear Schrödinger equations with power nonlinearity satisfying the gauge condition. The iterative use of the operator $x+i t \partial_{x}$ in $[\mathrm{H}-\mathrm{N}$ $\mathrm{T}$ ] allowed the authors to study the smoothing property of solutions to nonlinear Schrödinger equations satisfying the gauge condition.

In Section 5 we prove regularity in space of solutions to (NLS) by using regularity in time of solutions. However the proof in Section 5 is not applicable directly to the case of arbitrary dimension.

Our method in this paper can be applied to the system of nonlinear equations

$$
\left\{\begin{array}{l}
i \partial_{t} \tilde{u}+A \partial_{x}^{2} \tilde{u}=F(\tilde{u}) \\
\tilde{u}(0)=\tilde{u}_{0}
\end{array}\right.
$$

where

$$
\tilde{u}=\left(\begin{array}{l}
u \\
v
\end{array}\right), \quad A=\left(\begin{array}{ll}
a_{11} & a_{12} \\
a_{21} & a_{22}
\end{array}\right), \quad F(\tilde{u})=\left(\begin{array}{l}
f_{1}(u, v) \\
f_{2}(u, v)
\end{array}\right) \quad \text { and } \quad \tilde{u}_{0}=\left(\begin{array}{l}
u_{0} \\
v_{0}
\end{array}\right) .
$$

When

$$
\left(\begin{array}{ll}
a_{11} & a_{12} \\
a_{21} & a_{22}
\end{array}\right)=\left(\begin{array}{cc}
0 & -1 \\
-1 & 0
\end{array}\right), \quad\left(\begin{array}{l}
f_{1}(u, v) \\
f_{2}(u, v)
\end{array}\right)=\left(\begin{array}{c}
-i v \\
0
\end{array}\right)
$$

(1.4) is written as

$$
\left\{\begin{array}{l}
i \partial_{t} u-(\Delta v-v)=0 \\
i \partial_{t} v-\Delta u=0 \\
u(0)=u_{0}, v(0)=v_{0}
\end{array}\right.
$$

which is equivalent to the (linearized) Boussinesq equation

$$
\left\{\begin{array}{l}
\partial_{t}^{2} u+\partial_{x}^{4} u-\partial_{x}^{2} u=0 \\
u(0)=u_{0}, \quad u_{t}(0)=i\left(-\partial_{x}^{2}+1\right)^{-1} v_{0}
\end{array}\right.
$$


We can obtain the same result as that of Theorem 1.2 for (1.4) since the operator $P=x \partial_{x}+2 t \partial_{t}$ has the commutation relation

$$
\left[P, i \partial_{t}+A \partial_{x}^{2}\right]=2\left(i \partial_{t}+A \partial_{x}^{2}\right)
$$

Our method is also applicable to a diagonal system

$$
\left\{\begin{array}{l}
\partial_{t} \omega^{k}+\partial_{x}^{2 j+1} \omega^{k}+Q_{k}\left(\omega^{1}, \cdots, \omega^{m}, \partial_{x} \omega^{1}, \cdots, \partial_{x}^{2 j-1} \omega^{m}\right)=0 \\
(k=1, \cdots, m=m(j))
\end{array}\right.
$$

where $Q_{k}^{\prime} s$ are polynomials having no constants or linear terms, since the operator $x \partial_{x}+(2 j+1) t \partial_{t}$ almost commutes with the linear part of (1.5) and the local smoothing property for the linear part of (1.5) proved in [Ke-P-V 1,Section 4] works well for (1.5) thanks to the nonlinear terms $Q_{k}^{\prime} s$ which are independent of the derivatives of order $2 j$. The system (1.5) was studied in [Ke-P-V 3] to obtain local well-posedness of the initial value problem for higher order nonlinear dispersive equations of the form

$$
\left\{\begin{array}{l}
\partial_{t} u+\partial_{x}^{2 j+1} u+P\left(u, \partial_{x} u, \cdots, \partial_{x}^{2 j} u\right)=0 \\
u(0)=u_{0}
\end{array}\right.
$$

where $P$ is a polynomial having no constant or linear terms. We notice here that $P$ has the highest derivative $\partial_{x}^{2 j} u$. By using a gauge transformation we can write (1.6) as a diagonal system (1.5) (see [Ke-P-V 3] for details), and so local well posedness of (1.6) can be treated.

If we add a smallness condition on the data, our method can apply to a system

$$
\partial_{t} u^{k}+\partial_{x}^{2 j+1} u^{k}+P_{k}\left(u^{1}, \cdots, u^{m}, \cdots, \partial_{x}^{2 j} u^{1}, \cdots, \partial_{x}^{2 j} u^{m}\right)
$$

by using the sharp inhomogeneous version of local smoothing property given in [Ke-P-V 3, Theorem 2.1 (2.2)].

The main tool in the proofs of our main results is a first order differential operator which almost commutes with the linear part of the target nonlinear evolution equation and which has only derivatives with respect to the space variables for $t=0$ and has a derivative with respect to the time variable for $t \neq 0$. Hence the following question arises. For linear dispersive systems of the type

$$
\left\{\begin{array}{l}
\partial_{t} \tilde{u}+i P(D) \tilde{u}=0 \\
\tilde{u}(0)=\tilde{u}_{0}
\end{array}\right.
$$


where

$$
\tilde{u}=\left(\begin{array}{l}
u \\
v
\end{array}\right) \quad P(\xi)=\left(\begin{array}{ll}
p_{11}(\xi) & p_{12}(\xi) \\
p_{21}(\xi) & p_{22}(\xi)
\end{array}\right) \quad P(D)=\mathcal{F}^{-1} \mathcal{P}(\xi) \mathcal{F}
$$

do there exist such first order differential operators ?

Under some conditions on $P(D)$ which includes many applications in the theory of dispersive long waves of small amplitude, local smoothing effects for (1.7) was proved in [Co-Sau]. Laurey [L] studied the Cauchy problem for a third order nonlinear Schrödinger equations

$$
\left\{\begin{array}{l}
i \partial_{t} u+i a \partial_{x}^{3} u+b \partial_{x}^{2} u=\alpha|u|^{2} u+i \beta\left(\partial_{x}|u|^{2}\right) u+i \gamma|u|^{2} \partial_{x} u \\
u(0)=u_{0}
\end{array}\right.
$$

which is introduced by A. Hasegawa and Y. Kodama (see references cited in $[\mathrm{L}]$ ), where $a, b, \alpha, \beta, \gamma$ are given real parameters. The linear part of (1.8) satisfies the condition of [Co-Sau] and so it has the local smoothing effects. As can be seen in $[\mathrm{P}]$, the differential operator

$$
x-3 a t \partial_{x}^{2}-2 i b t \partial_{x}
$$

commutes with the linear part of (1.8). However it is difficult to construct a first order differential operator which almost commutes with the linear part of (1.8) from (1.9).

By combining the induction method in $[\mathrm{P}]$ and the local smoothing property for the linear part of (1.8) in [L, Proposition 2.1] we can obtain the similar result as $[\mathrm{P}$, Theorem 3.2] for (1.8). However the result in a Gevrey class function space is still open. More precisely, assuming that $u_{0}$ is in some Gevrey class, is the solution of (1.8) analytic in space variable or not?

This paper is organized as follows. In Section 2 we prove useful lemmas which are needed to obtain the main results. Section 3 is devoted to study the existence of solutions to $(\mathrm{gKdV})$ when the initial function is in some Gevrey class of order $\sigma$, which yields that the solution of ( $\mathrm{gKdV})$ is in the (usual) Gevrey class of order $\sigma$ in time variable. By using the existence results of Section 3, we prove Theorems 1.1 and 1.1' in Section 4. In Section 5 we state the results of [H-K.K, Propositions 3.3-3.4] that the solution of (NLS) is in the (usual) Gevrey class of order $\sigma$ in time when the initial function is in some Gevrey class of order $\sigma$ and using these results we show Theorems 1.2 and 1.2'. 


\section{PRELIMINARIES}

In Sections 2 and 3, we denote positive constants by $C$ and $C$ may change from line to line. We let $S(t)$ be the unitary group associated with the linear equation $\partial_{t} u+\partial_{x}^{3} u=0$. We first state well known estimates and local smoothing property of $S(t) \phi$ obtained by [Ke-P-V 1].

LEMMA 2.1. - For any $\phi \in L^{2}$ and any $t>0$

$$
\begin{gathered}
\|S(t) \phi\|_{L^{2}}=\|\phi\|_{L^{2}} \\
\sup _{x} \int_{\mathbb{R}}\left|\partial_{x} S(t) \phi(x)\right|^{2} d t \leq C\|\phi\|_{L^{2}}^{2}
\end{gathered}
$$

and for any $\phi \in L^{1}$,

$$
\|S(t) \phi\|_{L^{\infty}} \leq C(1+|t|)^{-\frac{1}{3}}\|\phi\|_{L^{1}}
$$

LEMMA 2.2. - We have for $P=x \partial_{x}+3 t \partial_{t}$

$$
\partial_{x} P^{l}=\sum_{k=0}^{l}\left(\begin{array}{l}
l \\
k
\end{array}\right) P^{l-k} \partial_{x}, \quad P^{l} \partial_{x}=\sum_{k=0}^{l}\left(\begin{array}{l}
l \\
k
\end{array}\right)(-1)^{k} \partial_{x} P^{l-k},
$$

$$
\partial_{t} P^{l}=\sum_{k=0}^{l}\left(\begin{array}{l}
l \\
k
\end{array}\right) 3^{k} P^{l-k} \partial_{t}, \quad P^{l} \partial_{t}=\sum_{k=0}^{l}\left(\begin{array}{l}
l \\
k
\end{array}\right)(-3)^{k} \partial_{t} P^{l-k}
$$

Proof. - We prove (2.1) and (2.2) by induction. When $l=1$, it is clear that the first equality of (2.1) is valid. We assume that the first one of (2.1) holds true for any $l$. Then we have by assumption

$$
\begin{aligned}
\partial_{x} P^{l+1} & =\left(P \partial_{x}+\partial_{x}\right) P^{l} \\
& =(P+1) \partial_{x} P^{l}=(P+1) \sum_{k=0}^{l}\left(\begin{array}{l}
l \\
k
\end{array}\right) P^{l-k} \partial_{x}
\end{aligned}
$$


By a direct calculation the right hand side of (2.3) equals

$$
\begin{aligned}
\sum_{k=0}^{l}\left(\begin{array}{l}
l \\
k
\end{array}\right)\left(P^{l+1-k} \partial_{x}+P^{l-k} \partial_{x}\right) & \\
\quad= & \sum_{k=1}^{l}\left(\begin{array}{l}
l \\
k
\end{array}\right) P^{l+1-k} \partial_{x}+\left(\begin{array}{l}
l \\
0
\end{array}\right) P^{l+1} \partial_{x}+\sum_{k=0}^{l}\left(\begin{array}{l}
l \\
k
\end{array}\right) P^{l-k} \partial_{x} \\
& =\left(\sum_{k=0}^{l-1}\left(\begin{array}{c}
l \\
k+1
\end{array}\right)+\sum_{k=0}^{l}\left(\begin{array}{l}
l \\
k
\end{array}\right)\right) P^{l-k} \partial_{x}+\left(\begin{array}{l}
l \\
0
\end{array}\right) P^{l+1} \partial_{x} \\
& =\sum_{k=0}^{l-1}\left(\left(\begin{array}{c}
l \\
k+1
\end{array}\right)+\left(\begin{array}{l}
l \\
k
\end{array}\right)\right) P^{l-k} \partial_{x}+\partial_{x}+P^{l+1} \partial_{x} \\
& =\sum_{k=0}^{l-1}\left(\begin{array}{c}
l+1 \\
k+1
\end{array}\right) P^{l-k} \partial_{x}+\partial_{x}+P^{l+1} \partial_{x} \\
& =\sum_{k=0}^{l}\left(\begin{array}{c}
l+1 \\
k+1
\end{array}\right) P^{l-k} \partial_{x}+P^{l+1} \partial_{x} \\
& =\sum_{k=1}^{l+1}\left(\begin{array}{c}
l+1 \\
k
\end{array}\right) P^{l+1-k} \partial_{x}+P^{l+1} \partial_{x}=\sum_{k=0}^{l+1}\left(\begin{array}{c}
l+1 \\
k
\end{array}\right) P^{l+1-k} \partial_{x} .
\end{aligned}
$$

From (2.3) and (2.4) we have

$$
\partial_{x} P^{l+1}=\sum_{k=0}^{l+1}\left(\begin{array}{c}
l+1 \\
k
\end{array}\right) P^{l+1-k} \partial_{x} .
$$

Hence we obtain the first part of (2.1). The second part of (2.1) and (2.2) are obtained in the same way as in the proof of the first equality of (2.1).

Q.E.D.

Lemma 2.3. - We let $\sigma \geq 1$ and $P=x \partial_{x}+3 t \partial_{t}$. Then we have

$$
\begin{aligned}
& \sum_{l=0}^{\infty} \frac{A^{l}}{(l !)^{\sigma}}\left\|\partial_{x} P^{l} g\right\|_{X} \leq e^{A} \sum_{l=0}^{\infty} \frac{A^{l}}{(l !)^{\sigma}}\left\|P^{l} \partial_{x} g\right\|_{X}, \\
& \sum_{l=0}^{\infty} \frac{A^{l}}{(l !)^{\sigma}}\left\|P^{l} \partial_{x} g\right\|_{X} \leq e^{A} \sum_{l=0}^{\infty} \frac{A^{l}}{(l !)^{\sigma}}\left\|\partial_{x} P^{l} g\right\|_{X}, \\
& \sum_{l=0}^{\infty} \frac{A^{l}}{(l !)^{\sigma}}\left\|\partial_{t} P^{l} g\right\|_{X} \leq e^{3 A} \sum_{l=0}^{\infty} \frac{A^{l}}{(l !)^{\sigma}}\left\|P^{l} \partial_{t} g\right\|_{X},
\end{aligned}
$$


and

$$
\sum_{l=0}^{\infty} \frac{A^{l}}{(l !)^{\sigma}}\left\|P^{l} \partial_{t} g\right\|_{X} \leq e^{3 A} \sum_{l=0}^{\infty} \frac{A^{l}}{(l !)^{\sigma}}\left\|\partial_{t} P^{l} g\right\|_{X}
$$

Proof. - Since every inequality in the lemma is proved in the same way by using Lemma 2.2 , we only prove the third one. By the first equality of (2.2) in Lemma 2.2 we have

$$
\begin{aligned}
& \sum_{l=0}^{\infty} \frac{A^{l}}{(l !)^{\sigma}}\left\|\partial_{t} P^{l} g\right\|_{X} \\
& \quad \leq \sum_{l=0}^{\infty} \frac{A^{l}}{(l !)^{\sigma}} \sum_{k=0}^{l}\left(\begin{array}{l}
l \\
k
\end{array}\right) 3^{k}\left\|P^{l-k} \partial_{t} g\right\|_{X} \\
& \quad \leq \sum_{l=0}^{\infty} \sum_{k=0}^{l}\left(\begin{array}{l}
l \\
k
\end{array}\right)^{1-\sigma} \frac{A^{l-k}}{((l-k) !)^{\sigma}} \frac{(3 A)^{k}}{(k !)^{\sigma}}\left\|P^{l-k} \partial_{t} g\right\|_{X} \\
& \quad \leq \sum_{l=0}^{\infty} \sum_{k=0}^{l} \frac{(3 A)^{k}}{(k !)^{\sigma}} \frac{A^{l-k}}{((l-k) !)^{\sigma}}\left\|P^{l-k} \partial_{t} g\right\|_{X} \\
& \quad \leq\left(\sum_{k=0}^{\infty} \frac{(3 A)^{k}}{(k !)^{\sigma}}\right)\left(\begin{array}{l}
\text { by } \\
\left(\sum_{l=0}^{\infty} \frac{A^{l}}{(l !)^{\sigma}}\left\|P^{l} \partial_{t} g\right\|_{X}\right.
\end{array}\right) \\
& \quad \leq e^{3 A} \sum_{l=0}^{\infty} \frac{A^{l}}{(l !)^{\sigma}}\left\|P^{l} \partial_{t} g\right\| x .
\end{aligned}
$$

Q.E.D.

Lemma 2.4. - We have

$$
\begin{aligned}
& \int_{-T}^{T}\|f(t) g(t)\|_{L^{2}} d t \\
& \quad \leq C T^{1 / 2} \sup _{x \in \mathbb{R}}\left(\int_{-T}^{T}|g(t, x)|^{2} d t\right)^{1 / 2}\left(\int_{\mathbb{R}} \sup _{t \in[-T, T]}|f(t, x)|^{2} d x\right)^{1 / 2},
\end{aligned}
$$

provided that the right hand side is finite. 
Proof. - By Schwarz' inequality we have

$$
\begin{aligned}
& \int_{-T}^{T}\|f(t) g(t)\|_{L^{2}} d t \leq C\left(\int_{-T}^{T}\|f(t) g(t)\|_{L^{2}}^{2} d t\right)^{1 / 2} T^{1 / 2} \\
& \quad=C\left(\int_{\mathbb{R}} \int_{-T}^{T}|f(t, x) g(t, x)|^{2} d t d x\right)^{1 / 2} T^{1 / 2} \\
& \quad \leq C\left(\int_{\mathbb{R}} \int_{-T}^{T}|g(t, x)|^{2} d t \cdot \sup _{t \in[-T, T]}|f(t, x)|^{2} d x\right)^{1 / 2} T^{1 / 2}
\end{aligned}
$$

from which the lemma follows.

In the same way as in the proof of Lemma 2.4 we have

LEMMA 2.4'.

$$
\begin{aligned}
\left(\int_{\mathbb{R}}\|f(t) g(t)\|_{L^{2}}^{2} d t\right)^{1 / 2} \leq & C \sup _{x \in \mathbb{R}}\left(\int_{\mathbb{R}}|g(t, x)|^{2} d t\right)^{1 / 2} \\
& \times\left(\int_{\mathbb{R}} \sup _{t \in \mathbb{R}}|f(t, x)|^{2} d x\right)^{1 / 2},
\end{aligned}
$$

provided that the right hand side is finite.

In order to prove Lemmas 2.5-2.5' we need [H-K.K, Proposition 2.1] and since we also use it in several stages of the proofs of the results in Section 3, we state the proposition without proof.

[H-K.K, Proposition 2.1.]. - We let

$$
f, g \in G_{\sigma}^{A_{1} A_{2}}\left(P, \partial_{x} ; H^{m}\right) \cap G_{\sigma}^{A_{1} A_{2}}\left(P, \partial_{x} ; H^{[m / 2], \infty}\right), \quad \sigma \geq 1
$$

and $P=x \partial_{x}+3 t \partial_{t}$. Then we have

$$
\begin{aligned}
& \|f \cdot g\|_{G_{\sigma}^{A_{1} A_{2}}\left(P+C_{1}, \partial_{x} ; H^{m}\right)} \\
& \leq e^{A_{1} C_{1}}\left(\|f\|_{G_{\sigma}^{A_{1} A_{2}}\left(P, \partial_{x} ; H^{[m / 2], \infty}\right)}\|g\|_{G_{\sigma}^{A_{1} A_{2}}\left(P, \partial_{x} ; H^{m}\right)}\right. \\
& \left.\quad+\|g\|_{G_{\sigma}^{A_{1} A_{2}}\left(P, \partial_{x} ; H^{[m / 2], \infty}\right)}\|f\|_{G_{\sigma}^{A_{1} A_{2}}\left(P, \partial_{x} ; H^{m}\right)}\right)
\end{aligned}
$$

where $[s]$ is the largest integer less than or equal to $s$.

The following lemma is needed to show the local existence in time of solutions to $(\mathrm{gKdV})$. 
Lemma 2.5. - We let $f \in Y_{T}, F(f)=f^{p-1} \partial_{x} f, \sigma \geq 1$ and $P=x \partial_{x}+3 t \partial_{t}$. Then we have

$$
\begin{aligned}
& \int_{0}^{T}\left(\|F(f)\|_{G_{\sigma}^{A_{1} A_{2}}\left(P, \partial_{x} ; H^{3}\right)}+\left\|\partial_{t} F(f)\right\|_{G_{\sigma}^{A_{1} A_{2}}\left(P, \partial_{x} ; L^{2}\right)} d \tau\right. \\
& \quad \leq C\left(T \mid\|f\|\left\|_{Y_{T}}^{p}+T^{1 / 2}\right\|\|f\|\left\|_{Y_{T}}\right\| f(0) \|_{G_{\sigma}^{A_{1} A_{2}}\left(x \partial_{x}, \partial_{x} ; H^{3}\right)}^{p-1}\right)
\end{aligned}
$$

Proof. - We have by lemma 2.3

$$
\text { (2.5) } \begin{aligned}
\|F(f)\|_{G_{\sigma}^{A_{1} A_{2}}\left(P, \partial_{x} ; H^{3}\right)} & =\sum_{l, k=0}^{\infty} \frac{A_{1}^{l}}{(l !)^{\sigma}} \frac{A_{2}^{k}}{(k !)^{\sigma}} \sum_{j=0}^{3}\left\|\partial_{x}^{j} P^{l} \partial_{x}^{k} F(f)\right\|_{L^{2}} \\
& \leq e^{3 A} \sum_{l, k=0}^{\infty} \frac{A_{1}^{l}}{(l !)^{\sigma}} \frac{A_{2}^{k}}{(k !)^{\sigma}} \sum_{j=0}^{3}\left\|P^{l} \partial_{x}^{k} \partial_{x}^{j} F(f)\right\|_{L^{2}} \\
& \leq e^{3 A} \sum_{j=0}^{3}\left\|\partial_{x}^{j} F(f)\right\|_{G_{\sigma}^{A_{1} A_{2}}\left(P, \partial_{x} ; L^{2}\right)} .
\end{aligned}
$$

A direct calculation gives

$$
\left\{\begin{aligned}
\partial_{x} F(f)= & (p-1) f^{p-2}\left(\partial_{x} f\right)^{2}+f^{p-1} \partial_{x}^{2} f \\
\partial_{x}^{2} F(f)= & (p-1)(p-2) f^{p-3}\left(\partial_{x} f\right)^{3} \\
& +3(p-1) f^{p-2} \partial_{x} f \cdot \partial_{x}^{2} f+f^{p-1} \partial_{x}^{3} f \\
\partial_{x}^{3} F(f)= & (p-1)(p-2)(p-3) f^{p-4}\left(\partial_{x} f\right)^{4} \\
& +6(p-1)(p-2) f^{p-3}\left(\partial_{x} f\right)^{2} \partial_{x}^{2} f \\
& +3(p-1) f^{p-2}\left(\partial_{x}^{2} f\right)^{2}+(p-1) f^{p-2} \partial_{x} f \cdot \partial_{x}^{3} f \\
& +f^{p-1} \partial_{x}^{4} f .
\end{aligned}\right.
$$

By the same argument as in the proof of [H-K.K, Proposition 2.1], Lemma 2.3 and Sobolev's inequality, $G_{\sigma}^{A_{1} A_{2}}\left(P, \partial_{x} ; L^{2}\right)$ norm of each term in the right hand side of (2.6) is estimated from above by

$$
C\left(\|f\|_{G_{\sigma}^{A_{1} A_{2}}\left(P, \partial_{x} ; H^{3}\right)}^{p}+\left\|f^{p-1} \partial_{x}^{4} f\right\|_{G_{\sigma}^{A_{1} A_{2}}\left(P, \partial_{x} ; L^{2}\right)}\right) .
$$

Hence we have by (2.7)

$$
\begin{aligned}
& \int_{0}^{T}\|F(f)\|_{G_{\sigma}^{A_{1} A_{2}}\left(P, \partial_{x} ; H^{3}\right)} d \tau \\
& \quad \leq C\left(T\|\| f\|\|_{Y_{T}}^{p}+\int_{0}^{T}\left\|f^{p-1} \partial_{x}^{4} f\right\|_{G_{\sigma}^{A_{1} A_{2}}\left(P, \partial_{x} ; L^{2}\right)} d \tau\right) .
\end{aligned}
$$


By Lemma 2.4 and a simple calculation

$\int_{0}^{T}\left\|g_{1} \cdot g_{2}\right\|_{G_{\sigma}^{A_{1} A_{2}}\left(P, \partial_{x} ; L^{2}\right)} d \tau$

$=\sum_{l, k=0}^{\infty} \frac{A_{1}^{l}}{(l !)^{\sigma}} \frac{A_{2}^{k}}{(k !)^{\sigma}}$

$\times \int_{0}^{T}\left\|P^{l} \partial_{x}^{k}\left(g_{1} \cdot g_{2}\right)\right\|_{L^{2}} d \tau$

$=\sum_{l, k=0}^{\infty} \frac{A_{1}^{l}}{(l !)^{\sigma}} \frac{A_{2}^{k}}{(k !)^{\sigma}} \sum_{j=0}^{l} \sum_{n=0}^{k}\left(\begin{array}{l}l \\ j\end{array}\right)\left(\begin{array}{l}k \\ n\end{array}\right)$

$\times \int_{0}^{T}\left\|P^{l-j} \partial_{x}^{k-n} g_{1} \cdot P^{j} \partial_{x}^{n} g_{2}\right\|_{L^{2}} d \tau$

$\leq \sum_{l, k=0}^{\infty} \frac{A_{1}^{l}}{(l !)^{\sigma}} \frac{A_{2}^{k}}{(k !)^{\sigma}} \sum_{j=0}^{l} \sum_{n=0}^{k}\left(\begin{array}{l}l \\ j\end{array}\right)\left(\begin{array}{l}k \\ n\end{array}\right) T^{1 / 2} \sup _{x \in \mathbf{R}}\left(\int_{0}^{T}\left|P^{j} \partial_{x}^{n} g_{2}\right|^{2} d \tau\right)^{1 / 2}$

$\times\left(\int_{\mathbb{R}} \sup _{t \in[0, T]}\left|P^{l-j} \partial_{x}^{k-n} g_{1}\right|^{2} d x\right)^{1 / 2}$

$\leq T^{1 / 2}\left(\sum_{l, k=0}^{\infty} \frac{A_{1}^{l}}{(l !)^{\sigma}} \frac{A_{2}^{k}}{(k !)^{\sigma}} \sup _{x \in R}\left(\int_{0}^{T}\left|P^{l} \partial_{x}^{k} g_{2}\right|^{2} d \tau\right)^{1 / 2}\right)$

$\times\left(\sum_{l, k=0}^{\infty} \frac{A_{1}^{l}}{(l !)^{\sigma}} \frac{A_{2}^{k}}{(k !)^{\sigma}}\left(\int_{\mathbb{R}} \sup _{t \in[0, T]}\left|P^{l} \partial_{x}^{k} g_{1}\right|^{2} d x\right)^{1 / 2}\right)$.

Since

$$
\left|g_{3}(t)\right|^{2}=\left|g_{3}(0)\right|^{2}+\int_{0}^{t} \partial_{\tau}\left|g_{3}(\tau)\right|^{2} d \tau
$$

we have by (2.9)

$$
\begin{aligned}
\int_{0}^{T} & \left\|g_{1} \cdot g_{2}\right\|_{G_{\sigma}^{A_{1} A_{2}}\left(P, \partial_{x} ; L^{2}\right)} d \tau \\
\leq & T^{1 / 2}\left(\sum_{l, k=0}^{\infty} \frac{A_{1}^{l}}{(l !)^{\sigma}} \frac{A_{2}^{k}}{(k !)^{\sigma}} \sup _{x \in \mathrm{R}}\left(\int_{0}^{T}\left|P^{l} \partial_{x}^{k} g_{2}\right|^{2} d \tau\right)^{1 / 2}\right) \\
& \times\left(\sum _ { l , k = 0 } ^ { \infty } \frac { A _ { 1 } ^ { l } } { ( l ! ) ^ { \sigma } } \frac { A _ { 2 } ^ { k } } { ( k ! ) ^ { \sigma } } \left(\left(\int_{\mathbb{R}}\left|\left(x \partial_{x}\right)^{l} \partial_{x}^{k} g_{1}(0, x)\right|^{2} d x\right)^{1 / 2}\right.\right. \\
& \left.+2\left(\int_{\mathbb{R}} \int_{0}^{T}\left|\partial_{\tau} P^{l} \partial_{x}^{k} g_{1}(\tau, x)\right|\left|P^{l} \partial_{x}^{k} g_{1}(\tau, x)\right| d \tau d x\right)^{1 / 2}\right)
\end{aligned}
$$




$$
\begin{aligned}
\leq & T^{1 / 2}\left(\sum_{l, k=0}^{\infty} \frac{A_{1}^{l}}{(l !)^{\sigma}} \frac{A_{2}^{k}}{(k !)^{\sigma}} \sup _{x \in \mathbb{R}}\left(\int_{0}^{T}\left|P^{l} \partial_{x}^{k} g_{2}\right|^{2} d \tau\right)^{1 / 2}\right) \\
& \times\left(\left\|g_{1}(0)\right\|_{G_{\sigma}^{A_{1} A_{2}}}\left(x \partial_{x}, \partial_{x} ; L^{2}\right)\right. \\
& \left.+T^{1 / 2} \gamma\left(T, P, 0, g_{1}\right)^{1 / 2} \gamma\left(T, P, 0, \partial_{t} g_{1}\right)^{1 / 2}\right)
\end{aligned}
$$

by Schwarz' inequality and Lemma 2.3 .

We apply (2.10) with $g_{1}=f^{p-1}$ and $g_{2}=\partial_{x}^{4} f$, and Lemma 2.3 to the right hand side of $(2.8)$ to obtain

$$
\begin{aligned}
\int_{0}^{T} & \|F(f)\|_{G_{\sigma}^{A_{1} A_{2}}\left(P, \partial_{x} ; H^{3}\right)} d \tau \\
\leq & C T\|\| f \|_{Y_{T}}^{p}+C T^{1 / 2} \sigma(T, P, 3, f) \\
& \times\left(\left\|f(0)^{p-1}\right\|_{G_{\sigma}^{A_{1} A_{2}}\left(x \partial_{x}, \partial_{x} ; L^{2}\right)}\right. \\
& \left.+T^{1 / 2} \gamma\left(T, P, 0, f^{p-1}\right)^{1 / 2} \gamma\left(T, P, 0, \partial_{t} f^{p-1}\right)^{1 / 2}\right)
\end{aligned}
$$

By using [H-K.K, Proposition 2.1] and Sobolev's inequality in the second term of the right hand side of (2.11) we get

$$
\begin{aligned}
& \int_{0}^{T}\|F(f)\|_{G_{\sigma}^{A_{1} A_{2}}\left(P, \partial_{x} ; H^{3}\right)} d \tau \\
& \quad \leq C\left(T \| \| f \| _ { Y _ { T } } ^ { p } + T ^ { 1 / 2 } \| \| f \| _ { Y _ { T } } \left(\|f(0)\|_{G_{\sigma}^{A_{1} A_{2}}\left(x \partial_{x}, \partial_{x} ; H^{3}\right)}^{p-1}\right.\right. \\
& \left.\left.\quad+T^{1 / 2}\|\| f \|\left.\right|_{Y_{T}} ^{p-1}\right)\right) \\
& \quad \leq C\left(T\|\| f\left\|_{Y_{T}}^{p}+T^{1 / 2}\right\|\|f\|_{Y_{T}}\|f(0)\|_{G_{\sigma}^{A_{1} A_{2}}\left(x \partial_{x}, \partial_{x} ; H^{3}\right)}^{p-1}\right)
\end{aligned}
$$

In the same way as in the proof of (2.8) we have

$$
\begin{aligned}
& \int_{0}^{T}\left\|\partial_{t} F(f)\right\|_{G_{\sigma}^{A_{1} A_{2}}\left(P, \partial_{x} ; L^{2}\right)} d \tau \\
& \quad \leq C\left(T\|\| f\left\|_{Y_{T}}^{p}+\int_{0}^{T}\right\| f^{p-1} \partial_{t} \partial_{x} f \|_{G_{\sigma}^{A_{1} A_{2}}\left(P, \partial_{x} ; L^{2}\right)} d \tau\right) .
\end{aligned}
$$

We again use (2.10) with $g_{1}=f^{p-1}$ and $g_{2}=\partial_{t} \partial_{x} f$, and Lemma 2.3 to Vol. $12, n^{\circ}$ 6-1995. 
the right hand side of the above. Then we have

$$
\begin{aligned}
\int_{0}^{T} & \left\|\partial_{t} F(f)\right\|_{G_{\sigma}^{A_{1} A_{2}}\left(P, \partial_{x} ; L^{2}\right)} d \tau \\
\leq & C T\|\| f \|_{Y_{T}}^{p}+C T^{1 / 2} \sigma\left(T, P, 0, \partial_{t} f\right) \\
& \times\left(\left\|f(0)^{p-1}\right\|_{G_{\sigma}^{A_{1} A_{2}}\left(x \partial_{x}, \partial_{x} ; L^{2}\right)}\right. \\
& \left.+T^{1 / 2} \gamma\left(T, P, 0, f^{p-1}\right)^{1 / 2} \gamma\left(T, P, 0, \partial_{t}\left(f^{p-1}\right)\right)^{1 / 2}\right)
\end{aligned}
$$

By the same argument as in the proof of (2.12), (2.13) yields

(2.14) $\int_{0}^{T}\left\|\partial_{t} F(f)\right\|_{G_{\sigma}^{A_{1} A_{2}}\left(P, \partial_{x} ; L^{2}\right)} d \tau \leq$ The right hand side of (2.12).

The lemma follows from (2.12) and (2.14) immediately.

In the same way as in the proof of Lemma 2.5 we have

Q.E.D.

LEMMA 2.6. - We let $F, \sigma, A_{1}$ and $P$ be the same as those in Lemma 2.5, and we let $f, g \in Y_{T}$ and $f(0)=g(0)$. Then we have

$$
\begin{aligned}
& \int_{0}^{T}\left(\|F(f)-F(g)\|_{G_{\sigma}^{A_{1} A_{2}}\left(P, \partial_{x} ; H^{3}\right)}+\left\|\partial_{t}(F(f)-F(g))\right\|_{G_{\sigma}^{A_{1} A_{2}}\left(P, \partial_{x} ; L^{2}\right)}\right) d \tau \\
& \quad \leq C T\|\| f-g\|\|_{Y_{T}}\left(\|\| f\left\|_{Y_{T}}^{p-1}+\right\|\|g\|_{Y_{T}}^{p-1}\right) .
\end{aligned}
$$

The following lemma is needed to show the global existence in time of solutions to $(\mathrm{gKdV})$.

LEMMA 2.5'. - We let $f \in Z_{\infty}, F(f)=f^{p-1} \partial_{x} f, \sigma \geq 1$ and $P=x \partial_{x}+3 t \partial_{t}$, where $p$ is an integer satisfying $p \geq 6$. Then we have

$$
\begin{gathered}
\int_{\mathbf{R}}\left(\|F(f)\|_{G_{\sigma}^{A_{1} A_{2}}\left(P, \partial_{x} ; H^{3}\right)}+\left\|\partial_{t} F(f)\right\|_{G_{\sigma}^{A_{1} A_{2}}\left(P, \partial_{x} ; L^{2}\right)}\right) d \tau \\
\leq C\left(\left.\|f\|\left\|_{Z_{\infty}}^{p}+\right\|\|f\|\right|_{Z_{\infty}} ^{3}\|f(0)\|_{G_{\sigma}^{A_{1} A_{2}}\left(x \partial_{x}, \partial_{x} ; H^{3}\right)}^{p-3}\right) .
\end{gathered}
$$


Proof. - We have by (2.5), (2.6) and [H-K.K, Proposition 2.1]

$$
\begin{aligned}
& \|F(f)\|_{G_{\sigma}^{A_{1} A_{2}}\left(P, \partial_{x} ; H^{3}\right)} \\
& \leq e^{3 A_{1}} \sum_{j=0}^{3}\left\|\partial_{x}^{j} F(f)\right\|_{G_{\sigma}^{A_{1} A_{2}}\left(P, \partial_{x} ; L^{2}\right)} \\
& \leq C\left(\|f\|_{G_{\sigma}^{A_{1} A_{2}}\left(P, \partial_{x} ; H^{1, \infty}\right)}^{p-1}\|f\|_{G_{\sigma}^{A_{1} A_{2}}\left(P, \partial_{x} ; H^{3}\right)}\right. \\
& \quad+\|f\|_{G_{\sigma}^{A_{1} A_{2}}\left(P, \partial_{x} ; L^{\infty}\right)}^{p-2}\left\|\left(\partial_{x}^{2} f\right)^{2}\right\|_{G_{\sigma}^{A_{1} A_{2}}\left(P, \partial_{x} ; L^{2}\right)} \\
& \left.\quad+\left\|f^{p-1} \partial_{x}^{4} f\right\|_{G_{\sigma}^{A_{1} A_{2}}\left(P, \partial_{x} ; L^{2}\right)}\right) \\
& \leq C\left(\|f\|_{G_{\sigma}^{A_{1} A_{2}}\left(P, \partial_{x} ; H^{1, \infty}\right)}^{p-2}\|f\|_{G_{\sigma}^{A_{1} A_{2}}\left(P, \partial_{x} ; H^{3}\right)}^{2}+\left\|f^{p-1} \partial_{x}^{4} f\right\|_{G_{\sigma}^{A_{1} A_{2}}\left(P, \partial_{x} ; L^{2}\right)}\right)
\end{aligned}
$$

(by Sobolev's inequality and Lemma 2.3).

Hence

$$
\begin{aligned}
\int_{\mathbf{R}}\|F(f)\|_{G_{\sigma}^{A_{1} A_{2}}\left(P, \partial_{x} ; H^{3}\right)} d \tau \\
\leq C \int_{\mathbf{R}}\|f\|_{G_{\sigma}^{A_{1} A_{2}}\left(P, \partial_{x} ; H^{1, \infty}\right)}^{p-2}\|f\|_{G_{\sigma}^{A_{1} A_{2}}\left(P, \partial_{x} ; H^{3}\right)}^{2} d \tau \\
\quad+C \int_{\mathbf{R}}\left\|f^{p-1} \partial_{x}^{4} f\right\|_{G_{\sigma}^{A_{1} A_{2}}\left(P, \partial_{x} ; L^{2}\right)} d \tau \\
\leq C \gamma(\infty, P, 3, f)^{2}\|f\|_{Z_{\infty}}^{p-2} \int_{\mathbf{R}}(1+|\tau|)^{-\frac{p-2}{3}} d \tau \\
\quad+C \int_{\mathbf{R}}\left\|f^{p-1} \partial_{x}^{4} f\right\|_{G_{\sigma}^{A_{1} A_{2}}\left(P, \partial_{x} ; L^{2}\right)} d \tau
\end{aligned}
$$

and since $(p-2) / 3>1$, the integral in the preceeding term is convergent and we get

$$
\begin{aligned}
& \int_{\mathbf{R}}\|F(f)\|_{G_{\sigma}^{A_{1} A_{2}\left(P, \partial_{x} ; H^{3}\right)}} d \tau \\
& \quad \leq C\left(\|\| f\left\|_{Z_{\infty}}^{p}+\int_{\mathbf{R}}\right\| f^{p-1} \partial_{x}^{4} f \|_{G_{\sigma}^{A_{1} A_{2}}\left(P, \partial_{x} ; L^{2}\right)} d \tau\right) .
\end{aligned}
$$

We have

$$
\begin{aligned}
& \int_{\mathbf{R}}\left\|f^{p-1} \partial_{x}^{4} f\right\|_{G_{\sigma}^{A_{1} A_{2}}\left(P, \partial_{x} ; L^{2}\right)} d \tau \\
& \quad \leq \int_{\mathbf{R}}\|f\|_{G_{\sigma}^{A_{1} A_{2}}\left(P, \partial_{x} ; L^{\infty}\right)}^{2}\left\|f^{p-3} \partial_{x}^{4} f\right\|_{G_{\sigma}^{A_{1} A_{2}}\left(P, \partial_{x} ; L^{2}\right)} d \tau
\end{aligned}
$$

(by [H-K.K, Proposition 2.1]) 


$$
\begin{aligned}
\leq & \int_{\mathbf{R}}\|f\|_{G_{\sigma}^{A_{1} A_{2}}\left(P, \partial_{x} ; L^{\infty}\right)}^{2} \\
& \times \sum_{l, k=0}^{\infty} \frac{A_{1}^{l}}{(l !)^{\sigma}} \frac{A_{2}^{k}}{(k !)^{\sigma}} \sum_{j=0}^{l} \sum_{n=0}^{k}\left(\begin{array}{l}
l \\
j
\end{array}\right)\left(\begin{array}{l}
k \\
n
\end{array}\right) \\
& \times\left\|P^{l-j} \partial_{x}^{k-n}\left(f^{p-3}\right) \cdot P^{j} \partial_{x}^{n} \partial_{x}^{4} f\right\|_{L^{2}} d \tau \\
\leq & \sum_{l, k=0}^{\infty} \frac{A_{1}^{l}}{(l !)^{\sigma}} \frac{A_{2}^{k}}{(k !)^{\sigma}} \sum_{j=0}^{l} \sum_{n=0}^{k}\left(\begin{array}{l}
l \\
j
\end{array}\right)\left(\begin{array}{l}
k \\
n
\end{array}\right) \\
& \times\left(\int_{\mathbf{R}}\|f\|_{G_{\sigma}^{A} A_{2}}^{4}\left(P, \partial_{x} ; L^{\infty}\right)\right. \\
& d \tau)^{1 / 2} \\
& \times\left(\int_{\mathbf{R}}\left\|P^{l-j} \partial_{x}^{k-n}\left(f^{p-3}\right) \cdot P^{j} \partial_{x}^{n} \partial_{x}^{4} f\right\|_{L^{2}}^{2} d \tau\right)^{1 / 2}
\end{aligned}
$$

(by Schwarz' inequality)

$$
\begin{aligned}
\leq & C \sum_{l, k=0}^{\infty} \frac{A_{1}^{l}}{(l !)^{\sigma}} \frac{A_{2}^{k}}{(k !)^{\sigma}} \sum_{j=0}^{l} \sum_{n=0}^{k}\left(\begin{array}{l}
l \\
j
\end{array}\right)\left(\begin{array}{l}
k \\
n
\end{array}\right)\|\| f \mid \|_{Z_{\infty}}^{2} \\
& \times \sup _{x \in \mathbf{R}}\left(\int_{\mathbf{R}}\left|P^{j} \partial_{x}^{n} \partial_{x}^{4} f\right|^{2} d t\right)^{1 / 2} \\
& \times\left(\int_{\mathbf{R}} \sup _{t \in \mathbb{R}}\left|P^{l-j} \partial_{x}^{k-n}\left(f^{p-3}\right)\right|^{2} d x\right)^{1 / 2} \quad \text { (by Lem }
\end{aligned}
$$$$
\leq\|\| f \|_{Z_{\infty}}^{2}\left(\sum_{l, k=0}^{\infty} \frac{A_{1}^{l}}{(l !)^{\sigma}} \frac{A_{2}^{k}}{(k !)^{\sigma}} \sup _{x \in \mathbf{R}}\left(\int_{\mathbf{R}}\left|P^{l} \partial_{x}^{k} \partial_{x}^{4} f\right|^{2} d t\right)^{1 / 2}\right)
$$$$
\times\left(\sum_{l, k=0}^{\infty} \frac{A_{1}^{l}}{(l !)^{\sigma}} \frac{A_{2}^{k}}{(k !)^{\sigma}}\left(\int_{\mathbf{R}} \sup _{t \in \mathbf{R}}\left|P^{l} \partial_{x}^{k}\left(f^{p-3}\right)\right|^{2} d x\right)^{1 / 2}\right)
$$$$
\leq\|f\| \|_{Z_{\infty}}^{3} \sum_{l, k=0}^{\infty} \frac{A_{1}^{l}}{(l !)^{\sigma}} \frac{A_{2}^{k}}{(k !)^{\sigma}}\left(\int_{\mathbf{R}} \sup _{t \in \mathbf{R}}\left|P^{l} \partial_{x}^{k}\left(f^{p-3}\right)\right|^{2} d x\right)^{1 / 2}
$$

(by Lemma 2.3).

In the same way as in the proof of Lemma 2.5 we use the fact that

$$
\begin{aligned}
\sup _{t \in \mathbf{R}}\left|P^{l} \partial_{x}^{k}\left(f^{p-3}\right)\right|^{2} \leq & \left|\left(x \partial_{x}\right)^{l} \partial_{x}^{k}\left(f(0)^{p-3}\right)\right|^{2} \\
& +\int_{\mathbf{R}}\left|P^{l} \partial_{x}^{k}\left(f^{p-3}\right)\right|\left|\partial_{t} P^{l} \partial_{x}^{k}\left(f^{p-3}\right)\right| d t
\end{aligned}
$$


to obtain

$$
\begin{aligned}
& \sum_{l, k=0}^{\infty} \frac{A_{1}^{l}}{(l !)^{\sigma}} \frac{A_{2}^{k}}{(k !)^{\sigma}}\left(\int_{\mathbf{R}} \sup _{t \in \mathbb{R}}\left|P^{l} \partial_{x}^{k}\left(f^{p-3}\right)\right|^{2} d x\right)^{1 / 2} \\
\leq & \left.\sum_{l, k=0}^{\infty} \frac{A_{1}^{l}}{(l !)^{\sigma}} \frac{A_{2}^{k}}{(k !)^{\sigma}}\left(\int_{\mathbf{R}} \mid\left(x \partial_{x}\right)^{l} \partial_{x}^{k}\left(f(0)^{p-3}\right)\right)\right|^{2} d x \\
+ & \left.C \int_{\mathbf{R}} \int_{\mathbf{R}}\left|\partial_{t} P^{l} \partial_{x}^{k}\left(f^{p-3}\right)\right|\left|P^{l} \partial_{x}^{k}\left(f^{p-3}\right)\right| d t d x\right)^{1 / 2} \\
\leq & C\left\|f(0)^{p-3}\right\|_{G_{\sigma}^{A_{1}} A_{2}}\left(x \partial_{x}, \partial_{x} ; L^{2}\right) \\
+ & C \sum_{l, k=0}^{\infty} \frac{A_{1}^{l}}{(l !)^{\sigma}} \frac{A_{2}^{k}}{(k !)^{\sigma}}\left(\int_{\mathbf{R}}\left(\int_{\mathbf{R}}\left|P^{l} \partial_{x}^{k}\left(f^{p-3}\right)\right|^{2} d x\right)^{1 / 2}\right. \\
\times & \left.\left(\int_{\mathbf{R}}\left|\partial_{t} P^{l} \partial_{x}^{k}\left(f^{p-3}\right)\right|^{2} d x\right)^{1 / 2} d t\right)^{1 / 2}
\end{aligned}
$$

(by Schwarz' inequality),

and by the same arguments as in [H-K.K, Proposition 2.1],

$$
\begin{aligned}
& \leq C\|f(0)\|_{G_{\sigma}^{A_{1} A_{2}}\left(x \partial_{x}, \partial_{x} ; H^{3}\right)}^{p-3} \\
& +C\left(\int_{\mathbf{R}}\|f\|_{G_{\sigma}^{A_{1} A_{2}}\left(P, \partial_{x} ; L^{\infty}\right)}^{p-4}\|f\|_{G_{\sigma}^{A_{1} A_{2}}\left(P, \partial_{x} ; L^{2}\right)}\right. \\
& \left.\times\left\|\partial_{t}\left(f^{p-3}\right)\right\|_{G_{\sigma}^{A_{1} A_{2}}\left(P, \partial_{x} ; L^{2}\right)} d t\right)^{1 / 2} \\
& \leq C\|f(0)\|_{G_{\sigma}^{A_{1} A_{2}}\left(x \partial_{x}, \partial_{x} ; H^{3}\right)}^{p-3} \\
& +C\left(\int_{\mathbf{R}}\|f\|_{G_{\sigma}^{A_{1} A_{2}}\left(P, \partial_{x} ; L \infty\right)}^{2(p-4)}\|f\|_{G_{\sigma}^{A_{1} A_{2}}\left(P, \partial_{x} ; L^{2}\right)}\right. \\
& \left.\times\left\|\partial_{t} f\right\|_{G_{\sigma}^{A_{1} A_{2}}\left(P, \partial_{x} ; L^{2}\right)} d t\right)^{1 / 2} \\
& \leq C\|f(0)\|_{G_{\sigma}^{A_{1} A_{2}}\left(x \partial_{x}, \partial_{x} ; H^{3}\right)}^{p-3} \\
& +C\|\| f \|_{Z_{\infty}}^{p-4} \gamma(\infty, P, 0, f)^{1 / 2} \gamma\left(\infty, P, 0, \partial_{t} f\right)^{1 / 2} .
\end{aligned}
$$

Vol. $12, n^{\circ}$ 6-1995. 
Hence we have

$$
\begin{aligned}
& \sum_{l, k=0}^{\infty} \frac{A_{1}^{l}}{(l !)^{\sigma}} \frac{A_{2}^{k}}{(k !)^{\sigma}}\left(\int_{\mathbb{R}} \sup _{t \in \mathbb{R}}\left|P^{l} \partial_{x}^{k}\left(f^{p-3}\right)\right|^{2} d x\right)^{1 / 2} \\
& \leq C\left(\|f(0)\|_{G_{\sigma}^{A_{1} A_{2}\left(x \partial_{x}, \partial_{x} ; H^{3}\right)}}^{p-3}+\|f\| \|_{Z_{\infty}}^{p-3}\right) .
\end{aligned}
$$

From (2.15), (2.16) and (2.17) it follows that

$$
\begin{aligned}
& \int_{\mathbb{R}}\|F(f)\|_{G_{\sigma}^{A_{1} A_{2}}\left(P, \partial_{x} ; H^{3}\right)} d t \\
& \quad \leq C\left(\|\| f\left\|_{Z_{\infty}}^{p}+\right\|\|f\|\left\|_{Z_{\infty}}^{3}\right\| f(0) \|_{G_{\sigma}^{A_{1} A_{2}}\left(x \partial_{x}, \partial_{x} ; H^{3}\right)}^{p-3}\right) .
\end{aligned}
$$

On the other hand, using the fact that

$$
\partial_{t} F(f)=f^{p-1} \partial_{t} \partial_{x} f+(p-1) f^{p-2} \partial_{t} f \cdot \partial_{x} f
$$

and the same arguments as in the proof of $(2.15)$, we can easily show that

$$
\begin{aligned}
& \int_{\mathbf{R}}\left\|\partial_{t} F(f)\right\|_{G_{\sigma}^{A_{1} A_{2}}\left(P, \partial_{x} ; L^{2}\right)} d t \\
& \quad \leq C\left(\|\| f\left\|_{Z_{\infty}}^{p}+\int_{\mathbf{R}}\right\| f^{p-1} \partial_{t} \partial_{x} f \|_{G_{\sigma}^{A_{1} A_{2}}\left(P, \partial_{x} ; L^{2}\right)} d t\right)
\end{aligned}
$$

In the same way as we proved (2.16), we have

$$
\begin{aligned}
& \int_{\mathbf{R}}\left\|f^{p-1} \partial_{t} \partial_{x} f\right\|_{G_{\sigma}^{A_{1} A_{2}}\left(P, \partial_{x} ; L^{2}\right)} d t \\
& \quad \leq\|\| f \|_{Z_{\infty}}^{3}\left(\sum_{l, k=0}^{\infty} \frac{A_{1}^{l}}{(l !)^{\sigma}} \frac{A_{2}^{k}}{(k !)^{\sigma}}\left(\int_{\mathbf{R}} \sup _{t \in \mathbb{R}}\left|P^{l} \partial_{x}^{k}\left(f^{p-3}\right)\right|^{2} d x\right)\right)^{1 / 2}
\end{aligned}
$$

and this, together with (2.19) and (2.17), gives

$$
\begin{aligned}
& \int_{\mathbf{R}}\left\|\partial_{t} F(f)\right\|_{G_{\sigma}^{A_{1} A_{2}}\left(P, \partial_{x} ; L^{2}\right)} d t \\
& \quad \leq C\left(\|f\|\left\|_{Z_{\infty}}^{p}+\right\|\|f\|\left\|_{Z_{\infty}}^{3}\right\| f(0) \|_{G_{\sigma}^{A_{1} A_{2}}\left(x \partial_{x}, \partial_{x} ; H^{3}\right)}^{p-3}\right) .
\end{aligned}
$$

From (2.18) and (2.19) the lemma follows. 
The following three lemmas are needed to show analyticity of solutions to $(\mathrm{gKdV})$ in space variable when the solutions are in a Gevrey class of order 3 in time variable.

LEMMA 2.7. - We have for any $m \in \mathbb{N}$

$$
\sum_{l^{\prime}=0}^{l}\left(\begin{array}{l}
l \\
l^{\prime}
\end{array}\right)^{-m} \leq 3
$$

Proof. - By an elementary calculation

$$
\sum_{l^{\prime}=0}^{l}\left(\begin{array}{l}
l \\
l^{\prime}
\end{array}\right)^{-m}=2+\sum_{l^{\prime}=1}^{l-1}\left(\begin{array}{l}
l \\
l^{\prime}
\end{array}\right)^{-m} \leq 2+\left(\frac{l-1}{l}\right)^{m} \leq 3 .
$$

Q.E.D.

LEMMA 2.8. - We have

$$
\left(\begin{array}{c}
l \\
l^{\prime}
\end{array}\right)\left(\begin{array}{c}
m \\
m^{\prime}
\end{array}\right)\left(\begin{array}{c}
l+m \\
l^{\prime}+m^{\prime}
\end{array}\right)^{-1} \leq 1 \text { for } l^{\prime} \leq l, m^{\prime} \leq m .
$$

Proof. - We expand both sides of the equality $(1+t)^{l+m}=(1+t)^{l}(1+t)^{m}$. Then we have

$$
\begin{gathered}
(1+t)^{l+m}=\sum_{k=0}^{l+m}\left(\begin{array}{c}
l+m \\
k
\end{array}\right) t^{k} \\
(1+t)^{l}(1+t)^{m}=\left(\sum_{k_{1}=0}^{l}\left(\begin{array}{c}
l \\
k_{1}
\end{array}\right) t^{k_{1}}\right)\left(\sum_{k_{2}=0}^{m}\left(\begin{array}{l}
m \\
k_{2}
\end{array}\right) t^{k_{2}}\right) \\
=\sum_{k_{1}=0}^{l} \sum_{k_{2}=0}^{m}\left(\begin{array}{c}
l \\
k_{1}
\end{array}\right)\left(\begin{array}{c}
m \\
k_{2}
\end{array}\right) t^{k_{1}+k_{2}}=\sum_{k=0}^{l+m}\left(\sum_{\substack{k_{1}+k_{2}=k \\
0 \leq k_{1} \leq l \\
0 \leq k_{2} \leq m}}\left(\begin{array}{c}
l \\
k_{1}
\end{array}\right)\left(\begin{array}{c}
m \\
k_{2}
\end{array}\right)\right) t^{k} .
\end{gathered}
$$

Hence

$$
\sum_{\substack{k_{1}+k_{2}=k \\
0 \leq k_{2} \leq l \\
0 \leq k_{2} \leq m}}\left(\begin{array}{c}
l \\
k_{1}
\end{array}\right)\left(\begin{array}{l}
m \\
k_{2}
\end{array}\right)=\left(\begin{array}{c}
l+m \\
k
\end{array}\right) .
$$

Since every term of the left hand side is positive, we have the result.

Q.E.D. 
LEMMA 2.9. - We have

$$
\begin{gathered}
\left(\begin{array}{c}
3 m \\
3 m_{1}
\end{array}\right)\left(\begin{array}{c}
m \\
m_{1}
\end{array}\right)^{-3} \leq 3^{6} \frac{\left(\max \left(1, m_{1}\right)\right)^{2 / 3}\left(\max \left(1, m-m_{1}\right)\right)^{2 / 3}}{m^{2 / 3}} \\
\text { for } m \in \mathbb{N}, 0 \leq m_{1} \leq m
\end{gathered}
$$

Proof. - We first prove

$$
\frac{1}{3} m^{1 / 3} \leq a(m)^{-1} \leq b(m)^{-1} \leq 3 m^{1 / 3} \quad \text { for } \quad m \geq 1
$$

where

$$
a(m)=\prod_{k=1}^{m} \frac{3 k-1}{3 k}, \quad b(m)=\prod_{k=1}^{m} \frac{3 k-2}{3 k} .
$$

For $m=1,2$, it is clear that (2.20) is valid, and so we consider the case $m \geq 2$. We have

$$
\log \left(a(m)^{-1}\right)=\sum_{k=1}^{m} \log \left(\frac{3 k}{3 k-1}\right)=\sum_{k=1}^{m} \log \left(1+\frac{1}{3 k-1}\right)
$$

Since $x-x^{2} \leq \log (1+x) \leq x$ for $x \geq 0$, the above equality gives

$$
\sum_{k=1}^{m}\left(\frac{1}{3 k-1}-\frac{1}{(3 k-1)^{2}}\right) \leq \log \left(a(m)^{-1}\right) \leq \sum_{k=1}^{m} \frac{1}{3 k-1}
$$

By an elementary calculation

$$
\frac{1}{3} \int_{1}^{m} \frac{1}{x} d x \leq \sum_{k=1}^{m} \frac{1}{3 k-1} \leq \frac{1}{2}+\frac{1}{3} \int_{1}^{m} \frac{1}{x} d x
$$

from which it follows

$$
\frac{1}{3} \log m \leq \sum_{k=1}^{m} \frac{1}{3 k-1} \leq \frac{1}{2}+\frac{1}{3} \log m
$$

and so

$$
\log e^{-1 / 3} m^{1 / 3} \leq \log \left(a(m)^{-1}\right) \leq \log e^{1 / 2} m^{1 / 3} \quad \text { for } \quad m \geq e .
$$


This implies

$$
\frac{1}{3} m^{1 / 3} \leq a(m)^{-1} \leq 3 m^{1 / 3} \text { for } m \geq 3
$$

Similarly, we have

$$
\frac{1}{3} m^{1 / 3} \leq b(m)^{-1} \leq 3 m^{1 / 3} \text { for } \quad m \geq 3
$$

Therefore (2.20) comes from (2.21) and (2.22). We note that

$$
\frac{(3 m) !}{(m !)^{3}}=3^{3 m} a(m) b(m) \text {. }
$$

From this we see that if $m_{1} \neq 0, m_{1} \neq m$

$$
\begin{aligned}
& \left(\begin{array}{c}
3 m \\
3 m_{1}
\end{array}\right)\left(\begin{array}{c}
m \\
m_{1}
\end{array}\right)^{-3}=\frac{(3 m) !}{(m !)^{3}} \cdot \frac{\left(m_{1} !\right)^{3}}{\left(3 m_{1}\right) !} \cdot \frac{\left(\left(m-m_{1}\right) !\right)^{3}}{\left(3 m-3 m_{1}\right) !} \\
= & a(m) b(m) a\left(m_{1}\right)^{-1} b\left(m_{1}\right)^{-1} a\left(m-m_{1}\right)^{-1} b\left(m-m_{1}\right)^{-1} \\
\leq & 3^{6} m^{-2 / 3} m_{1}^{2 / 3}\left(m-m_{1}\right)^{2 / 3} .
\end{aligned}
$$

When $m_{1}=0$ or $m_{1}=m$,

$$
\left(\begin{array}{c}
3 m \\
3 m_{1}
\end{array}\right)\left(\begin{array}{c}
m \\
m_{1}
\end{array}\right)^{-3}=1
$$

From (2.23) and (2.24) the lemma follows.

Q.E.D.

The following lemma is needed to show analyticity in space variable of solutions to (NLS) which are in a Gevrey class of order 2 in time variable, and is proved in the same way as Lemma 2.9.

LEMMA 2.10. - We have

$$
\begin{gathered}
\left(\begin{array}{c}
2 m \\
2 m_{1}
\end{array}\right)\left(\begin{array}{c}
m \\
m_{1}
\end{array}\right)^{-2} \leq 2^{6} \frac{\left(\max \left(1, m_{1}\right)\right)\left(\max \left(1, m-m_{1}\right)\right)}{m^{1 / 2}} \\
\text { for } \quad m \in \mathbb{N}, 0 \leq m_{1} \leq m .
\end{gathered}
$$




\section{EXISTENCE OF SOLUTIONS TO (gKdV)}

In this section we prove

Proposition 3.1. - Let $\phi \in G_{\sigma}^{A_{1} A_{2}}\left(x \partial_{x}, \partial_{x}, H^{3}\right)$. Then there exist a unique solution $u$ of $(\mathrm{gKdV})$ and a positive constant $T$ such that $u \in Y_{T}$.

Proposition 3.1'. - In addition to the assumptions on Proposition 3.1, we assume that

$$
\begin{gathered}
\phi \in G_{\sigma}^{A_{1} A_{2}}\left(x \partial_{x}, \partial_{x} ; H^{1,1}\right), \\
\epsilon_{1}=\|\phi\|_{G_{\sigma}^{A_{1} A_{2}}\left(x \partial_{x}, \partial_{x} ; H^{1,1}\right)}+\|\phi\|_{G_{\sigma}^{A_{1} A_{2}}\left(x \partial_{x}, \partial_{x} ; H^{3}\right)}
\end{gathered}
$$

is sufficiently small and the nonlinear term satisfies the same growth conditions as those in Theorem 1.1'. Then there exists a unique global solution $u$ of $(g K d V)$ such that $u \in Z_{\infty}$.

In what follows we only consider positive time, since the case of negative time is treated similarly. We let $u_{n}$ be the solution of

$$
\left\{\begin{array}{l}
L u_{n}=\lambda u_{n-1}^{p-1} \partial_{x} u_{n-1} \equiv F\left(u_{n-1}\right), \\
u_{n}(0)=\phi
\end{array}\right.
$$

for $n \geq 1$, and $u_{0}$ be the solution of

$$
L u_{0}=0, \quad u_{0}(0)=\phi,
$$

where $L=\partial_{t}+\partial_{x}^{3}$. In the same way as in the proof of [H-K.K, (3.3)] we have by induction

$$
L \partial_{x}^{j} P^{l} \partial_{x}^{k} u_{n}(t)=\partial_{x}^{j}(P+3)^{l} \partial_{x}^{k} F\left(u_{n-1}(t)\right) .
$$

The integral equation associated with (3.3) is written as

$$
\begin{aligned}
\partial_{x}^{j} P^{l} \partial_{x}^{k} u_{n}(t)= & S(t) \partial_{x}^{j}\left(x \partial_{x}\right)^{l} \partial_{x}^{k} \phi \\
& +\int_{0}^{t} S(t-\tau) \partial_{x}^{j}(P+3)^{l} \partial_{x}^{k} F\left(u_{n-1}(\tau)\right) d \tau
\end{aligned}
$$

where $S(t)$ is the unitary group associated with the linear equation $L u=0$.

By using formula (3.4) and Lemma 2.1 we prove Propositions 3.1-3.1'.

Proof of Proposition 3.1. - It is sufficient to prove that $\left\{u_{n}\right\}$ is a Cauchy sequence in $Y_{T}$ when $T$ is sufficiently small. Taking $L^{2}$ norm in (3.4), 
multiplying both sides of the resulting inequality by $A_{1}^{l} A_{2}^{k} /(l !)^{\sigma}(k !)^{\sigma}$, making a summation with respect to $l$ and $k$, and using Lemma 2.1, we obtain

$$
\begin{aligned}
& \gamma\left(T, P, 3, u_{n}\right)+\sigma\left(T, P, 3, u_{n}\right) \\
& \quad \leq C\left(\|\phi\|_{Y_{0}}+\int_{0}^{T}\left\|F\left(u_{n-1}(\tau)\right)\right\|_{G_{\sigma}^{A_{1} A_{2}}\left(P+3, \partial_{x} ; H^{3}\right)} d \tau\right)
\end{aligned}
$$

where and in what follows we let for simplicity

$$
\|\cdot\|_{Y_{0}}=\|\cdot\|_{G_{\sigma}^{A_{1} A_{2}}\left(x \partial_{x}, \partial_{x} ; H^{3}\right)} \cdot
$$

In the same way as in the proof of (3.5) we have

$$
\begin{aligned}
\gamma\left(T, P, 0, \partial_{t} u_{n}\right)+\sigma\left(T, P, 0, \partial_{t} u_{n}\right) \\
\leq C\left(\left\|\partial_{t} u_{n}(0)\right\|_{Y_{0}}+\int_{0}^{T}\left\|\partial_{\tau} F\left(u_{n-1}(\tau)\right)\right\|_{G_{\sigma}^{A_{1} A_{2}}\left(P+3, \partial_{x} ; L^{2}\right)} d \tau\right) \\
\leq C\left(\|\phi\|_{Y_{0}}+\|F(\phi)\|_{G_{\sigma}^{A_{1} A_{2}}\left(x \partial_{x}, \partial_{x} ; L^{2}\right)}\right. \\
\left.\quad+\int_{0}^{T}\left\|\partial_{\tau} F\left(u_{n-1}(\tau)\right)\right\|_{G_{\sigma}^{A_{1} A_{2}}\left(P+3, \partial_{x} ; L^{2}\right)} d \tau\right) .
\end{aligned}
$$

We have by [H-K.K, Proposition 2.1] and Sobolev's inequality

$$
\|F(\phi)\|_{G_{\sigma}^{A_{1} A_{2}}\left(x \partial_{x}, \partial_{x} ; L^{2}\right)} \cdot \leq C\|\phi\|_{Y_{0}}^{p} .
$$

From (3.5)-(3.7) it follows that

$$
\begin{aligned}
\left.\left\|u_{n}\right\|\right|_{Y_{T}} \leq C & \left\{\|\phi\|_{Y_{0}}\left(1+\|\phi\|_{Y_{0}}^{p-1}\right)\right. \\
& +\int_{0}^{T}\left(\left\|F\left(u_{n-1}(\tau)\right)\right\|_{G_{\sigma}^{A_{1} A_{2}}\left(P+3, \partial_{x} ; H^{3}\right)}\right. \\
& \left.\left.+\left\|\partial_{\tau} F\left(u_{n-1}(\tau)\right)\right\|_{G_{\sigma}^{A_{1} A_{2}}\left(P+3, \partial_{x} ; L^{2}\right)}\right) d \tau\right\} \\
\leq C & \left\{\|\phi\|_{Y_{0}}\left(1+\|\phi\|_{Y_{0}}^{p-1}\right)\right.
\end{aligned}
$$




$$
\begin{aligned}
& +\int_{0}^{T}\left(\left\|F\left(u_{n-1}(\tau)\right)\right\|_{G_{\sigma}^{A_{1} A_{2}}\left(P, \partial_{x} ; H^{3}\right)}\right. \\
& \left.\left.+\left\|\partial_{\tau} F\left(u_{n-1}(\tau)\right)\right\|_{G_{\sigma}^{A_{1} A_{2}}\left(P, \partial_{x} ; L^{2}\right)}\right) d \tau\right\} \\
& \qquad(\text { by }[\mathrm{H}-\mathrm{K} . \mathrm{K}, \text { Lemma 2.1]) } \\
& \leq C\left\{\|\phi\|_{Y_{0}}\left(1+\|\phi\|_{Y_{0}}^{p-1}\right)+T\|\| u_{n-1} \|_{Y_{T}}^{p}\right. \\
& \left.+T^{1 / 2}\|\| u_{n-1}\left\|\left.\right|_{Y_{T}}\right\| \phi \|_{Y_{0}}^{p-1}\right\} \\
& \leq C\left\{\|\phi\|_{Y_{0}}\left(1+\|\phi\|_{Y_{0}}^{p-1}\right)\right. \\
& \left.+\left(T+T^{p / 2}\right)\left\|u_{n-1}\right\|_{Y_{T}}^{p}\right\} .
\end{aligned}
$$

From (3.2) and energy estimates it follows that

$$
\left\|u_{0}\right\|_{Y_{T}} \leq\|\phi\|_{Y_{0}}
$$

We take $\rho$ such that

$$
C\|\phi\| Y_{0}\left(1+\|\phi\|_{Y_{0}}^{p-1}\right) \leq \frac{\rho}{2}
$$

and $T$ such that

$$
C\left(T+T^{p / 2}\right) \leq \frac{1}{2}
$$

in the right hand side of (3.8). Then we have by (3.8) and (3.9)

$$
\left\|u_{n}\right\|_{Y_{T}} \leq \rho \text { for any } n \in \mathbb{N} .
$$

Proposition 3.1 is obtained by showing $\left\{u_{n}\right\}$ is a Cauchy sequence in $Y_{T}$. In the same way as in the proof of the second inequality of (3.8)

$$
\begin{aligned}
& \|\| u_{n+1}-u_{n} \|\left.\right|_{Y_{T}} \\
& \leq C \int_{0}^{T}\left(\left\|F\left(u_{n}\right)-F\left(u_{n-1}\right)\right\|_{G_{\sigma}^{A_{1} A_{2}}\left(P, \partial_{x} ; H^{3}\right)}\right. \\
& \left.\quad+\left\|\partial_{\tau}\left(F\left(u_{n}\right)-F\left(u_{n-1}\right)\right)\right\|_{G_{\sigma}^{A_{1} A_{2}}\left(P, \partial_{x} ; L^{2}\right)}\right) d \tau \\
& \leq\left. C T\left\|u_{n}-u_{n-1}\right\|\right|_{Y_{T}}\left(\left\|u_{n}\right\|_{Y_{T}^{p-1}}^{p-1}+\left\|u_{n-1}\right\| \|_{Y_{T}}^{p-1}\right) \\
& \quad \text { (by Lemma 2.6) } \\
& \leq C T \rho^{p-1}\|\| u_{n}-u_{n-1}\|\|_{Y_{T}}
\end{aligned}
$$

(by (3.10)). 
If we take $T$ satisfying

$$
C T \rho^{p-1} \leq \frac{1}{2}
$$

in the right hand side of (3.11) we have by (3.10)

$$
\left.\left\|\left|u_{n+1}-u_{n}\left\|\left.\right|_{Y_{T}} \leq \frac{1}{2}\left|\left\|u_{n}-u_{n-1}\right\|\right|_{Y_{T}} \leq\left(\frac{1}{2}\right)^{n}\right\|\right| u_{1}-u_{0}\right\|\right|_{Y_{T}} \leq\left(\frac{1}{2}\right)^{n} \rho .
$$

This means that $\left\{u_{n}\right\}$ is a Cauchy sequence in $Y_{T}$. This completes the proof of Proposition 3.1.

Q.E.D.

Proof of Proposition 3.1'. - In the same way as in the proof of the second inequality in (3.8) we obtain by (3.4)

$$
\begin{aligned}
\left\|u_{n}\right\|_{Y_{\infty}} \leq C & \left\{\|\phi\|_{Y_{0}}\left(1+\|\phi\|_{Y_{0}}^{p-1}\right)\right. \\
& +\int_{0}^{\infty}\left(\left\|F\left(u_{n-1}(t)\right)\right\|_{G_{\sigma}^{A_{1} A_{2}}\left(P, \partial_{x} ; H^{3}\right)}\right. \\
& \left.\left.+\left\|\partial_{t} F\left(u_{n-1}(t)\right)\right\|_{G_{\sigma}^{A_{1} A_{2}}\left(P, \partial_{x} ; L^{2}\right)}\right) d t\right\} .
\end{aligned}
$$

We apply Lemma $2.5^{\prime}$ to the above to have

$$
\begin{aligned}
& \|\| u_{n} \mid \| Y_{Y_{0}} \\
& \leq C\left\{\|\phi\|_{Y_{0}}\left(1+\|\phi\|_{Y_{0}}^{p-1}\right)+\|\| u_{n-1}\left\|_{Z_{\infty}}^{p}+\right\|\left\|u_{n-1}\right\|\left\|_{Z_{\infty}}^{3}\right\| \phi \|_{Y_{0}}^{p-3}\right\} \\
& \leq C\left\{\|\phi\|_{Y_{0}}\left(1+\|\phi\|_{Y_{0}}^{p-1}\right)+\|\| u_{n-1} \|\left.\right|_{Z_{\infty}} ^{p}\right\}
\end{aligned}
$$

(by Young's inequality).

On the other hand, by Lemma 2.1

$$
\|S(t) f\|_{H^{1, \infty}} \leq C(1+t)^{-1 / 3}\left(\|f\|_{H^{1,1}}+\|f\|_{H^{2}}\right) .
$$

Vol. 12, n 6-1995. 
Hence we have by (3.4) and [H-K.K, Propositions 2.1-2.3]

$$
\begin{aligned}
& \left\|u_{n}(t)\right\|_{G_{\sigma}^{A_{1} A_{2}}\left(P, \partial_{x} ; H^{1, \infty}\right)} \leq C \epsilon_{1}(1+t)^{-1 / 3}+C \int_{0}^{t}(1+(t-\tau))^{-1 / 3} \\
& \quad \times\left(\left\|F\left(u_{n-1}(\tau)\right)\right\|_{G_{\alpha}^{A_{1} A_{2}}\left(P+3, \partial_{x} ; H^{1,1}\right)}\right. \\
& \left.\quad+\left\|F\left(u_{n-1}(\tau)\right)\right\|_{G_{o}^{A_{1} A_{2}}\left(P+3, \partial_{x} ; H^{2}\right)}\right) d \tau \\
& \leq C\left(\epsilon_{1}(1+t)^{-1 / 3}+\|\| u_{n-1} \|_{Y_{\infty}}^{2} \int_{0}^{t}(1+(t-\tau))^{-1 / 3}\right. \\
& \left.\quad \times\left\|u_{n-1}(\tau)\right\|_{G_{\sigma}^{A_{1} A_{2}}\left(P, \partial_{x} ; H^{1, \infty}\right)}^{p-2} d \tau\right) \\
& \leq C\left(\epsilon_{1}(1+t)^{-1 / 3}+\|\| u_{n-1}\|\|_{Y_{\infty}}^{p} \int_{0}^{t}(1+(t-\tau))^{-\frac{1}{3}}(1+\tau)^{-\frac{p-2}{3}} d \tau\right)
\end{aligned}
$$

which gives

$$
(1+t)^{1 / 3}\left\|u_{n}(t)\right\|_{G_{\sigma}^{A_{1} A_{2}}\left(P, \partial_{x} ; H^{1, \infty}\right)} \leq C\left(\epsilon_{1}+\|\| u_{n-1}\|\|_{Z_{\infty}}^{p}\right) .
$$

By (3.12) and (3.13)

$$
\left\|u_{n}\right\| \|_{z_{\infty}} \leq C\left(\epsilon_{1}+\|\| u_{n-1}\|\|_{Z_{\infty}}^{p}\right)
$$

In the same way as in the proof of $(3.14)$ we have

$$
\left\|u_{0}\right\|_{z_{\infty}} \leq C \epsilon_{1}
$$

this with (3.14) gives

$$
\left\|u_{n}\right\| z_{\infty} \leq C \epsilon_{1}, \quad \text { for any } n \in \mathbb{N}
$$

Similarly, we have by Lemma $2.6^{\prime}$ and (3.15)

$$
\left.\left\||| u_{n+1}-u_{n}\right\|\right|_{z_{\infty}} \leq C \epsilon_{1}^{p-1} \mid\left\|u_{n}-u_{n-1}\right\| z_{z_{\infty}} \leq C\left(\frac{1}{2}\right)^{n} \epsilon_{1} .
$$

By (3.15) and (3.16) we have the result.

Q.E.D.

In the same way as in the proofs of [H-K.K, Proposition 3.3-3.4] we have by Proposition 3.1-3.1' 
Proposition 3.2. - Let $u$ be the solution of $(g K d V)$ constructed in Proposition 3.1. Then

$$
u \in G_{\sigma}^{|t| A_{3}}\left(\partial_{t} ; H^{3}(-R, R)\right) \text { for }|t| \leq T
$$

where

$$
A_{3} \leq \min \left\{\frac{3 A_{1}}{1+3 A_{1}}, \frac{3 A_{2}}{4 A_{2}+e^{1 / 3}(1+R)}\right\} .
$$

Proposition 3.2'. - Let $u$ be the solution of $(g K d V)$ constructed in Proposition 3.1'. Then

$$
u \in G_{\sigma}^{|t| A_{3}}\left(\partial_{t} ; H^{3}(-R, R)\right) \quad \text { for any } \quad t \in \mathbb{R}
$$

and

$$
\|u(t)\|_{G_{\sigma}^{|t| A_{3}}\left(\partial_{t} ; H^{1, \infty}(-R, R)\right)} \leq C \epsilon_{1}(1+|t|)^{-1 / 3} \quad \text { for any } \quad t \in \mathbb{R},
$$

where $R$ and $A_{3}$ are the same as in Proposition 3.2.

\section{PROOFS OF THEOREMS 1.1-1.1' (gKdV)}

By Propositions 3.1, 3.1', 3.2, 3.2', to obtain Theorems 1.1,1.1' it is sufficient to prove

Proposition 4.1. - Let $u$ be the solution of $(g K d V)$ satisfying

$$
u \in G_{\sigma}^{|t| A_{3}}\left(\partial_{t}, H^{3}(-R, R)\right) .
$$

Then there exists a positive constant $A_{4}$ such that

$$
u \in G_{\max (\sigma / 3,1)}^{|t|^{1 / 3} A_{4}}\left(\partial_{x} ; H^{3}(-R, R)\right) .
$$

Proof of Proposition 4.1. - We divide the proposition into the following two lemmas.

LEMMA 4.1. - Let $u$ be the solution of ( $g K d V)$ satisfying

$$
u \in G_{\sigma}^{|t| A_{3}}\left(\partial_{t}, H^{3}(-R, R)\right) .
$$

Then there exists a positive constant $A_{5}$ such that

$$
u \in G_{\max (\sigma, 3)}^{|t| A_{5}}\left(\partial_{x}^{3} ; H^{3}(-R, R)\right) .
$$

Vol. $12, \mathrm{n}^{\circ}$ 6-1995. 
LEMMA 4.2. - Let $u$ be a function satisfying

$$
u \in G_{\max (\sigma, 3)}^{|t| A_{5}}\left(\partial_{x}^{3} ; H^{3}(-R, R)\right) .
$$

Then there exists a positive constant $A_{4}$ such that

$$
u \in G_{\max (\sigma / 3,1)}^{|t|^{1 / 3} A_{4}}\left(\partial_{x} ; H^{3}(-R, R)\right) .
$$

Proof of Lemma 4.1. - It suffices to prove the result for $t=1$. By the assumption we have

$$
C_{0}=\sum_{l=0}^{\infty} \frac{A_{3}^{l}}{(l !)^{\sigma}}\left\|\partial_{t}^{l} u\right\|_{H^{3}(-R, R)}<\infty
$$

which implies

$$
\frac{A_{3}^{l}}{(l !)^{\sigma}}\left\|\partial_{t}^{l} u\right\|_{H^{3}(-R, R)} \leq C_{0}
$$

We prove by induction with respect to $m$ that there exists a positive constant $A_{6}$ such that

$$
\frac{A_{3}^{l+m}}{((l+m) !)^{\sigma^{l}}}\left\|\partial_{t}^{l} \partial_{x}^{3 m} u\right\|_{H^{1}(-R, R)} \leq C_{0} A_{6}^{m}(\max (1, m))^{-4 / 3}
$$

$$
\frac{A_{3}^{l+m}}{((l+m) !)^{\sigma^{\prime}}}\left\|\partial_{t}^{l} \partial_{x}^{3 m+k} u\right\|_{H^{1}(-R, R)} \leq A C_{0} A_{6}^{m}
$$

for $l, m \in \mathbb{N} \cup\{0\}, k=1,2$, where $\sigma^{\prime}=\max (\sigma, 3), A$ is a positive constant determined later. If (4.2) is valid, then taking $l=0$ in (4.2) and (4.3) we have Lemma 4.1 with

$$
A_{5}=\frac{A_{3}}{A_{6}}-\epsilon, \quad(\epsilon>0) .
$$

It is clear that (4.2) and (4.3) hold for all $l$ and $k=1,2$ when $m=0$. We assume that (4.2) and (4.3) are valid for all $l, m$ and $k=1,2$. For simplicity we denote

$$
\|\cdot\|_{H^{1}(-R, R)}=\|\cdot\|_{1}
$$

until the end of of the proof of Proposition 4.1 . 
Since

$$
\partial_{x}^{3} u=-\partial_{t} u-\lambda\left(\partial_{x}\left(u^{p}\right)\right) / p
$$

we have

$$
\partial_{t}^{l} M^{m+1} \partial_{x}^{k} u=-\partial_{t}^{l+1} M^{m} \partial_{x}^{k} u-\frac{\lambda}{p} \partial_{t}^{l} M^{m} \partial_{x}^{k+1}\left(u^{p}\right),
$$

where and in what follows

$$
M=\partial_{x}^{3}
$$

Hence

$$
\begin{aligned}
\left\|\partial_{t}^{l} M^{m+1} \partial_{x}^{k} u\right\|_{1} & \leq\left\|\partial_{t}^{l+1} M^{m} \partial_{x}^{k} u\right\|_{1}+|\lambda|\left\|\partial_{t}^{l} M^{m} \partial_{x}^{k}\left(u^{p-1} u_{x}\right)\right\|_{1} \\
& \equiv I_{1, k}+|\lambda| I_{2, k}
\end{aligned}
$$

The crucial term is $I_{2, k}$ because it is easy to handle $I_{1, k}$ by the induction assumption. Indeed we have

(4.5) $I_{1, k} \cdot \frac{A_{3}^{l+m+1}}{((l+m+1) !)^{\sigma^{\prime}}} \leq\left\{\begin{array}{l}C_{0} A_{6}^{m}(\max (1, m))^{-4 / 3} \\ A C_{0} A_{6}^{m} \text { for } k=1,2 .\end{array}\right.$ for $k=0$,

We shall prove that

(4.6) $|\lambda| I_{2, k} \cdot \frac{A_{3}^{l+m+1}}{((l+m+1) !)^{\sigma^{\prime}}} \leq\left\{\begin{array}{l}\frac{1}{2} C_{0} A_{6}^{m+1}(1+m)^{-4 / 3} \text { for } k=0, \\ \frac{1}{2} A C_{0} A_{6}^{m+1} \text { for } k=1,2 .\end{array}\right.$

For simplicity, we show (4.6) in the case $p=2$, since the general case $p \geq 3$ can be proved by induction. We have by Sobolev's inequality

$$
\begin{gathered}
\|\cdot\|_{L^{\infty}(-R, R)} \leq \frac{C}{4}\|\cdot\|_{H^{1}(-R, R)} \\
I_{2, k}=\left\|\partial_{t}^{l} \partial_{x}^{3 m+k}\left(u \cdot u_{x}\right)\right\|_{1} \\
\leq C \sum_{l^{\prime}=0}^{l} \sum_{m^{\prime}=0}^{3 m+k}\left(\begin{array}{c}
l \\
l^{\prime}
\end{array}\right)\left(\begin{array}{c}
3 m+k \\
m^{\prime}
\end{array}\right)\left\|\partial_{t}^{l^{\prime}} \partial_{x}^{m^{\prime}} u\right\|_{1}\left\|\partial_{t}^{l-l^{\prime}} \partial_{x}^{3 m+k+1-m^{\prime}} u\right\|_{1} .
\end{gathered}
$$


First we treat the term $I_{2.0}$. We have by (4.7)

$$
\text { (4.8) } \begin{aligned}
& \frac{1}{C} I_{2,0} \leq \sum_{l^{\prime}=0}^{l} \sum_{m^{\prime}=0}^{3 m}\left(\begin{array}{c}
l \\
l^{\prime}
\end{array}\right)\left(\begin{array}{c}
3 m \\
m^{\prime}
\end{array}\right)\left\|\partial_{t}^{l^{\prime}} \partial_{x}^{m^{\prime}} u\right\|_{1}\left\|\partial_{t}^{l-l^{\prime}} \partial_{x}^{3 m+1-m^{\prime}} u\right\|_{1} \\
= & \sum_{l^{\prime}=0}^{l} \sum_{m_{1}=0}^{m}\left(\begin{array}{c}
l \\
l^{\prime}
\end{array}\right)\left(\begin{array}{c}
3 m \\
3 m_{1}
\end{array}\right)\left\|\partial_{t}^{l^{\prime}} M^{m_{1}} u\right\|_{1}\left\|\partial_{t}^{l-l^{\prime}} M^{m-m_{1}} \partial_{x} u\right\|_{1} \\
& +\sum_{l^{\prime}=0}^{l} \sum_{m_{1}=0}^{m-1}\left(\begin{array}{c}
l \\
l^{\prime}
\end{array}\right)\left(\begin{array}{c}
3 m \\
3 m_{1}+1
\end{array}\right)\left\|\partial_{t}^{l^{\prime}} M^{m_{1}} \partial_{x} u\right\|_{1}\left\|\partial_{t}^{l-l^{\prime}} M^{m-m_{1}} u\right\|_{1} \\
& +\sum_{l^{\prime}=0}^{l} \sum_{m_{1}=0}^{m-1}\left(\begin{array}{c}
l \\
l^{\prime}
\end{array}\right)\left(\begin{array}{c}
3 m \\
3 m_{1}+2
\end{array}\right)\left\|\partial_{t}^{l^{\prime}} M^{m_{1}} \partial_{x}^{2} u\right\|_{1}\left\|\partial_{t}^{l-l^{\prime}} M^{m-m_{1}-1} \partial_{x}^{2} u\right\|_{1} \\
\equiv & I_{2,0,1}+I_{2,0,2}+I_{2,0,3} .
\end{aligned}
$$

Hence

$$
I_{2,0,1} \cdot \frac{A_{3}^{l+m+1}}{((l+m+1) !)^{\sigma^{\prime}}}
$$

$$
\leq \sum_{l^{\prime}=0}^{l} \sum_{m_{1}=0}^{m}\left(\begin{array}{c}
l \\
l^{\prime}
\end{array}\right)\left(\begin{array}{c}
3 m \\
3 m_{1}
\end{array}\right)\left(\begin{array}{c}
l+m \\
l^{\prime}+m_{1}
\end{array}\right)^{-\sigma^{\prime}} \cdot \frac{A_{3}}{(l+m+1)^{\sigma^{\prime}}}
$$$$
\times \frac{A_{3}^{l^{\prime}+m_{1}}}{\left(\left(l^{\prime}+m_{1}\right) !\right)^{\sigma^{\prime}}}\left\|\partial_{t}^{l^{\prime}} M^{m_{1}} u\right\|_{1} \frac{A_{3}^{l+m-l^{\prime}-m_{1}}}{\left(\left(l+m-l^{\prime}-m_{1}\right) !\right) \sigma^{\prime}}\left\|\partial_{t}^{l-l^{\prime}} M^{m-m_{1}} \partial_{x} u\right\|_{1}
$$$$
\leq \sum_{l^{\prime}=0}^{l} \sum_{m_{1}=0}^{m}\left(\begin{array}{c}
l \\
l^{\prime}
\end{array}\right)^{1-\sigma^{\prime}}\left(\begin{array}{c}
3 m \\
3 m_{1}
\end{array}\right)\left(\begin{array}{c}
m \\
m_{1}
\end{array}\right)^{-\sigma^{\prime}}\left[\left(\begin{array}{c}
l \\
l^{\prime}
\end{array}\right)\left(\begin{array}{c}
m \\
m_{1}
\end{array}\right)\left(\begin{array}{c}
l+m \\
l^{\prime}+m_{1}
\end{array}\right)^{-1}\right]^{\sigma^{\prime}}
$$$$
\times \frac{A_{3}}{(l+m+1)^{\sigma^{\prime}}} C_{0} A_{6}^{m_{1}}\left(\max \left(1, m_{1}\right)\right)^{-4 / 3} A C_{0} A_{6}^{m-m_{1}}
$$

(by the induction assumption)

$$
\leq 3 \sum_{m_{1}=0}^{m}\left(\begin{array}{c}
3 m \\
3 m_{1}
\end{array}\right)\left(\begin{array}{c}
m \\
m_{1}
\end{array}\right)^{-3} \frac{A_{3}}{(1+m)^{3}} A C_{0}^{2} A_{6}^{m}\left(\max \left(1, m_{1}\right)\right)^{-4 / 3}
$$

(by Lemmas 2.7-2.8) 


$$
\begin{aligned}
& \leq 3^{7} \sum_{m_{1}=0}^{m} \frac{\left(\max \left(1, m_{1}\right)\right)^{2 / 3}\left(\max \left(1, m-m_{1}\right)\right)^{2 / 3}}{m^{2 / 3}} \frac{A_{3}}{(1+m)^{3}} \\
& \times A C_{0}^{2} A_{6}^{m}\left(\max \left(1, m_{1}\right)\right)^{-4 / 3} \quad \text { (by Lemma 2.9) } \\
& \leq 3^{7} A C_{0}^{2} \sum_{m_{1}=0}^{m} \frac{\left(\max \left(1, m_{1}\right)\right)^{-2 / 3}\left(\max \left(1, m-m_{1}\right)\right)^{2 / 3}}{m^{2 / 3}} \frac{A_{3}}{(1+m)^{3}} A_{6}^{m} \\
& \leq 3^{7} A C_{0}^{2} \frac{A_{3}}{(1+m)^{2}} A_{6}^{m} .
\end{aligned}
$$

In the same way as in the proof of (4.9) we have

$$
I_{2,0,2} \cdot \frac{A_{3}^{l+m+1}}{((l+m+1) !)^{\sigma^{\prime}}}
$$

$$
\begin{aligned}
\leq & \sum_{l^{\prime}=0}^{l} \sum_{m_{1}=0}^{m-1}\left(\begin{array}{c}
l \\
l^{\prime}
\end{array}\right)^{1-\sigma^{\prime}}\left(\begin{array}{c}
3 m \\
3 m_{1}+1
\end{array}\right)\left(\begin{array}{c}
m \\
m_{1}
\end{array}\right)^{-\sigma^{\prime}}\left[\left(\begin{array}{c}
l \\
l^{\prime}
\end{array}\right)\left(\begin{array}{c}
m \\
m_{1}
\end{array}\right)\left(\begin{array}{c}
l+m \\
l^{\prime}+m_{1}
\end{array}\right)^{-1}\right]^{\sigma^{\prime}} \\
& \times \frac{A_{3}}{(l+m+1)^{\sigma^{\prime}}} A C_{0} A_{6}^{m_{1}}\left(\max \left(1, m_{1}\right)\right)^{-4 / 3} C_{0} A_{6}^{m-m_{1}}
\end{aligned}
$$

(by the induction assumption)

$$
\leq 3 \sum_{m_{1}=0}^{m-1}\left(\begin{array}{c}
3 m \\
3 m_{1}+1
\end{array}\right)\left(\begin{array}{c}
m \\
m_{1}
\end{array}\right)^{-3} \frac{A_{3}}{(1+m)^{3}} A C_{0}^{2} A_{6}^{m}\left(\max \left(1, m_{1}\right)\right)^{-4 / 3}
$$

(by Lemmas 2.7-2.8)

$$
=3 \sum_{m_{1}=0}^{m-1}\left(\begin{array}{c}
3 m \\
3 m_{1}
\end{array}\right)\left(\begin{array}{c}
m \\
m_{1}
\end{array}\right)^{-3} \frac{3\left(m-m_{1}\right)}{3 m_{1}+1} \frac{A_{3}}{(1+m)^{3}} A C_{0}^{2} A_{6}^{m}\left(\max \left(1, m_{1}\right)\right)^{-4 / 3}
$$

Vol. $12, \mathrm{n}^{\circ}$ 6-1995. 


$$
\begin{aligned}
\leq & 3^{8} A C_{0}^{2} \sum_{m_{1}=0}^{m-1} \frac{\left(\max \left(1, m_{1}\right)\right)^{2 / 3}\left(\max \left(1, m-m_{1}\right)\right)^{-1 / 3}}{m^{2 / 3}} \\
& \times \frac{A_{3}}{(1+m)^{3}} A_{6}^{m} \frac{1}{3 m_{1}+1}
\end{aligned}
$$

(by Lemma 2.9)

$$
\leq 3^{8} A C_{0}^{2} \frac{A_{3}}{(1+m)^{2}} A_{6}^{m}
$$

and we also have

$$
\begin{aligned}
I_{2,0,3} & \cdot \frac{A_{3}^{l+m+1}}{((l+m+1) !)^{\sigma^{\prime}}} \\
\leq & \sum_{l^{\prime}=0}^{l} \sum_{m_{1}=0}^{m-1}\left(\begin{array}{l}
l \\
l^{\prime}
\end{array}\right)\left(\begin{array}{c}
3 m \\
3 m_{1}+2
\end{array}\right)\left(\begin{array}{c}
l+m \\
l^{\prime}+m_{1}
\end{array}\right)^{-\sigma^{\prime}} \\
& \times \frac{A_{3}}{\left(l+m-l^{\prime}-m_{1}\right)^{\sigma^{\prime}}} \cdot \frac{A_{3}}{(l+m+1)^{\sigma^{\prime}}} \\
& \times \frac{A_{3}^{l^{\prime}+m_{1}}}{\left(\left(l^{\prime}+m_{1}\right) !\right)^{\sigma^{\prime}}}\left\|\partial_{t}^{l^{\prime}} M^{m_{1}} \partial_{x}^{2} u\right\|_{1} \\
& \times \frac{A_{3}^{l+m-l^{\prime}-m_{1}-1}}{\left(\left(l+m-l^{\prime}-m_{1}-1\right) !\right)^{\sigma^{\prime}}\left\|\partial_{t}^{l-l^{\prime}} M^{m-m_{1}-1} \partial_{x}^{2} u\right\|_{1}} \\
\leq & \sum_{l^{\prime}=0}^{l} \sum_{m_{1}=0}^{m-1}\left(\begin{array}{c}
l \\
l^{\prime}
\end{array}\right){ }^{1-\sigma^{\prime}}\left(\begin{array}{c}
3 m \\
3 m_{1}+2
\end{array}\right)\left(\begin{array}{c}
m \\
m
\end{array}\right)^{-\sigma^{\prime}} \\
& \times\left[\left(\begin{array}{c}
l \\
l^{\prime}
\end{array}\right)\left(\begin{array}{c}
m \\
m_{1}
\end{array}\right)\left(\begin{array}{c}
l+m \\
l^{\prime}+m_{1}
\end{array}\right)^{-1}\right]^{\sigma^{\prime}} \\
& \times \frac{A_{3}}{\left(l+m-l^{\prime}-m_{1}\right)^{\sigma^{\prime}} \cdot \frac{A_{3}}{\left(l+m^{\prime}+1\right)^{\sigma^{\prime}}} A^{2} C_{0}^{2} A_{6}^{m-1}}
\end{aligned}
$$

(by the induction assumption)

$$
\leq 3 \sum_{m_{1}=0}^{m-1}\left(\begin{array}{c}
3 m \\
3 m_{1}+2
\end{array}\right)\left(\begin{array}{c}
m \\
m_{1}
\end{array}\right)^{-3} \frac{A_{3}^{2}}{(1+m)^{3}\left(m-m_{1}\right)^{3}} A^{2} C_{0}^{2} A_{6}^{m-1}
$$

(by Lemmas 2.7-2.8) 


$$
\begin{aligned}
= & 3 \sum_{m_{1}=0}^{m-1}\left(\begin{array}{c}
3 m \\
3 m_{1}
\end{array}\right)\left(\begin{array}{c}
m \\
m_{1}
\end{array}\right)^{-3} \\
& \times \frac{3\left(m-m_{1}\right)\left(3 m-3 m_{1}-1\right)}{\left(3 m_{1}+1\right)\left(3 m_{1}+2\right)} \frac{A_{3}^{2} A^{2} C_{0}^{2} A_{6}^{m-1}}{(1+m)^{3}\left(m-m_{1}\right)^{3}} \\
& \quad \leq 3^{9} A^{2} C_{0}^{2} \frac{A_{3}^{2}}{(1+m)^{2}} A_{6}^{m-1} \quad \text { (by Lemma 2.9). }
\end{aligned}
$$

Consequently we have

$$
\begin{aligned}
& \frac{1}{C} I_{2,0} \cdot \frac{A_{3}^{l+m+1}}{((l+m+1) !)^{\sigma^{\prime}}} \\
& \leq\left(2 \cdot 3^{8} A C_{0}^{2} A_{3} A_{6}^{-1}+3^{9} A^{2} C_{0}^{2} A_{3}^{2} A_{6}^{-2}\right) \frac{1}{(m+1)^{2}} A_{6}^{m+1} .
\end{aligned}
$$

If we take

$$
\tilde{C}=C\left(2 \cdot 3^{8} A C_{0}^{2} A_{3} A_{6}^{-1}+3^{9} A^{2} C_{0}^{2} A_{3}^{2} A_{6}^{-2}\right) .
$$

Then

$$
|\lambda| I_{2,0} \cdot \frac{A_{3}^{l+m+1}}{((l+m+1) !)^{\sigma^{\prime}}} \leq|\lambda| \tilde{C} A_{6}^{m+1}(1+m)^{-4 / 3}
$$

which implies (4.6) for $k=0$ if

$$
\frac{1}{4} A_{6}=\max \left\{|\lambda| C 2 \cdot 3^{8} C_{0} A A_{3},\left(|\lambda| C \cdot 3^{9} C_{0}\right)^{1 / 2} A A_{3}\right\}
$$

Next we treat the case $k=1$. We have by (4.7)

$$
\begin{aligned}
\leq & \sum_{l^{\prime}=0}^{l} \sum_{m^{\prime}=0}^{3 m+1}\left(\begin{array}{c}
l \\
l^{\prime}
\end{array}\right)\left(\begin{array}{c}
3 m+1 \\
m^{\prime}
\end{array}\right)\left\|\partial_{t}^{l^{\prime}} \partial_{x}^{m^{\prime}} u\right\|_{1}\left\|\partial_{t}^{l-l^{\prime}} \partial_{x}^{3 m+2-m^{\prime}} u\right\|_{1} \\
= & \sum_{l^{\prime}=0}^{l} \sum_{m_{1}=0}^{m}\left(\begin{array}{c}
l \\
l^{\prime}
\end{array}\right)\left(\begin{array}{c}
3 m+1 \\
3 m_{1}
\end{array}\right)\left\|\partial_{t}^{l^{\prime}} M^{m_{1}} u\right\|_{1}\left\|\partial_{t}^{l-l^{\prime}} M^{m-m_{1}} \partial_{x}^{2} u\right\|_{1} \\
& +\sum_{l^{\prime}=0}^{l} \sum_{m_{1}=0}^{m}\left(\begin{array}{c}
l \\
l^{\prime}
\end{array}\right)\left(\begin{array}{c}
3 m+1 \\
3 m_{1}+1
\end{array}\right)\left\|\partial_{t}^{l^{\prime}} M^{m_{1}} \partial_{x} u\right\|_{1}\left\|\partial_{t}^{l-l^{\prime}} M^{m-m_{1}} \partial_{x} u\right\|_{1} \\
& +\sum_{l^{\prime}=0}^{l} \sum_{m_{1}=0}^{m-1}\left(\begin{array}{l}
l \\
l^{\prime}
\end{array}\right)\left(\begin{array}{c}
3 m+1 \\
3 m_{1}+2
\end{array}\right)\left\|\partial_{t}^{l^{\prime}} M^{m_{1}} \partial_{x}^{2} u\right\|_{1}\left\|\partial_{t}^{l-l^{\prime}} M^{m-m_{1}} u\right\|_{1} \\
\equiv & I_{2,1,1}+I_{2,1,2}+I_{2,1,3} .
\end{aligned}
$$

Vol. 12, $\mathrm{n}^{\circ}$ 6-1995. 
In the same way as in the proofs of (4.9)-(4.11) we have

$$
\begin{aligned}
& I_{2,1,1} \cdot \frac{A_{3}^{l+m+1}}{((l+m+1) !)^{\sigma^{\prime}}} \\
& \leq 3 \sum_{m_{1}=0}^{m}\left(\begin{array}{c}
3 m \\
3 m_{1}
\end{array}\right)\left(\begin{array}{c}
m \\
m_{1}
\end{array}\right)^{-3} A C_{0}^{2} A_{6}^{m} A_{3} \\
& \quad \times \frac{1}{(m+1)^{3}}\left(\max \left(1, m_{1}\right)\right)^{-4 / 3} \frac{3 m+1}{3 m-3 m_{1}+1} \\
& \leq 3^{8} A C_{0}^{2} A_{3} A_{6}^{m},
\end{aligned}
$$

(4.15) $\quad I_{2,1,2} \cdot \frac{A_{3}^{l+m+1}}{((l+m+1) !)^{\sigma^{\prime}}}$

$$
\begin{aligned}
& \leq 3 \sum_{m_{1}=0}^{m}\left(\begin{array}{c}
3 m \\
3 m_{1}
\end{array}\right)\left(\begin{array}{c}
m \\
m_{1}
\end{array}\right)^{-3} A^{2} C_{0}^{2} A_{6}^{m} A_{3} \frac{1}{(m+1)^{3}} \frac{3 m+1}{3 m_{1}+1} \\
& \leq 3^{8} A^{2} C_{0}^{2} A_{3} A_{6}^{m}
\end{aligned}
$$

(4.16) $\quad I_{2,1,3} \cdot \frac{A_{3}^{l+m+1}}{((l+m+1) !)^{\sigma^{\prime}}}$

$$
\begin{aligned}
\leq & 3 \sum_{m_{1}=0}^{m-1}\left(\begin{array}{c}
3 m \\
3 m_{1}
\end{array}\right)\left(\begin{array}{c}
m \\
m_{1}
\end{array}\right)^{-3} \\
& \times \frac{A C_{0}^{2} A_{6}^{m} A_{3}}{(m+1)^{3}}\left(\max \left(1, m-m_{1}\right)\right)^{-4 / 3} \frac{3 m+1}{3 m_{1}+1} \frac{3 m-3 m_{1}}{3 m_{1}+2} \\
\leq & 3^{9} A C_{0}^{2} A_{3} A_{6}^{m} .
\end{aligned}
$$

From (4.13)-(4.16), (4.6) follows for $k=1$ if

$$
\frac{1}{4} A_{6}=|\lambda| 3^{8} C C_{0} A_{3} \max \{6, A\}
$$


Finally we treat the case $k=2$. We have by (4.7)

$$
\begin{aligned}
& \frac{1}{C} I_{2,2} \\
\leq & \sum_{l^{\prime}=0}^{l} \sum_{m^{\prime}=0}^{3 m+2}\left(\begin{array}{c}
l \\
l^{\prime}
\end{array}\right)\left(\begin{array}{c}
3 m+2 \\
m^{\prime}
\end{array}\right)\left\|\partial_{t}^{l^{\prime}} \partial_{x}^{m^{\prime}} u\right\|_{1}\left\|\partial_{t}^{l-l^{\prime}} \partial_{x}^{3 m+2+1-m^{\prime}} u\right\|_{1} \\
= & \sum_{l^{\prime}=0}^{l} \sum_{m_{1}=0}^{m}\left(\begin{array}{c}
l \\
l^{\prime}
\end{array}\right)\left(\begin{array}{c}
3 m+2 \\
3 m_{1}
\end{array}\right)\left\|\partial_{t}^{l^{\prime}} M^{m_{1}} u\right\|_{1}\left\|\partial_{t}^{l-l^{\prime}} M^{m+1-m_{1}} u\right\|_{1} \\
& +\sum_{l^{\prime}=0}^{l} \sum_{m_{1}=0}^{m}\left(\begin{array}{c}
l \\
l^{\prime}
\end{array}\right)\left(\begin{array}{c}
3 m+2 \\
3 m_{1}+1
\end{array}\right)\left\|\partial_{t}^{l^{\prime}} M^{m_{1}} \partial_{x} u\right\|_{1}\left\|\partial_{t}^{l-l^{\prime}} M^{m-m_{1}} \partial_{x}^{2} u\right\|_{1} \\
& +\sum_{l^{\prime}=0}^{m} \sum_{m_{1}=0}^{m}\left(\begin{array}{c}
l \\
l^{\prime}
\end{array}\right)\left(\begin{array}{c}
3 m+2 \\
3 m_{1}+2
\end{array}\right)\left\|\partial_{t}^{l^{\prime}} M^{m_{1}} \partial_{x}^{2} u\right\|_{1}\left\|\partial_{t}^{l-l^{\prime}} M^{m-m_{1}} \partial_{x} u\right\|_{1} \\
\equiv & I_{2,2,1}+I_{2,2,2}+I_{2,2,3} .
\end{aligned}
$$

In the same way as in the proof of (4.9) we have by (4.18)

$$
\begin{aligned}
\text { (4.19) } & I_{2,2,1} \cdot \frac{A_{3}^{l+m+1}}{((l+m+1) !)^{\sigma^{\prime}}} \\
\leq & \sum_{l^{\prime}=0}^{l} \sum_{m_{1}=0}^{m}\left(\begin{array}{c}
l \\
l^{\prime}
\end{array}\right)\left(\begin{array}{c}
3 m+2 \\
3 m_{1}
\end{array}\right)\left(\begin{array}{c}
l+m+1 \\
l^{\prime}+m_{1}
\end{array}\right)^{-\sigma^{\prime}} \\
& \times \frac{A_{3}^{l^{\prime}+m_{1}}}{\left(\left(l^{\prime}+m_{1}\right) !\right)^{\sigma^{\prime}}}\left\|\partial_{t}^{l^{\prime}} M^{m_{1}} u\right\|_{1} \\
& \times \frac{A_{3}^{l+m+1-l^{\prime}-m_{1}}}{\left(\left(l+m+1-l^{\prime}-m_{1}\right) !\right)^{\sigma^{\prime}}\left\|\partial_{t}^{l-l^{\prime}} M^{m+1-m_{1}} u\right\|_{1}} \\
\leq & \sum_{l^{\prime}=0}^{l} \sum_{m_{1}=0}^{m}\left(\begin{array}{c}
l \\
l^{\prime}
\end{array}\right)^{1-\sigma^{\prime}}\left(\begin{array}{c}
3 m+2 \\
3 m_{1}
\end{array}\right)\left(\begin{array}{c}
m+1 \\
m_{1}
\end{array}\right)^{-\sigma^{\prime}} \\
& \times\left[\left(\begin{array}{c}
l \\
l^{\prime}
\end{array}\right)\left(\begin{array}{c}
m+1 \\
m_{1}
\end{array}\right)\left(\begin{array}{c}
l+m+1 \\
l^{\prime}+m_{1}
\end{array}\right)^{-1}\right]^{\sigma^{\prime}} \\
& \times C_{0} A_{6}^{m_{1}}\left(\max \left(1, m_{1}\right)\right)^{-4 / 3} C_{0} A_{6}^{m+1-m_{1}}\left(\max \left(1, m+1-m_{1}\right)\right)^{-4 / 3}
\end{aligned}
$$

(by the induction assumption) 


$$
\begin{aligned}
\leq & 3 \sum_{m_{1}=0}^{m}\left(\begin{array}{c}
3 m+2 \\
3 m_{1}
\end{array}\right)\left(\begin{array}{c}
m+1 \\
m_{1}
\end{array}\right)^{-3} C_{0}^{2} A_{6}^{m+1} \\
& \times\left(\max \left(1, m_{1}\right)\right)^{-4 / 3}\left(\max \left(1, m+1-m_{1}\right)\right)^{-4 / 3}
\end{aligned}
$$

(by Lemmas 2.7-2.8)

$$
\begin{aligned}
= & 3 \sum_{m_{1}=0}^{m}\left(\begin{array}{c}
3(m+1) \\
3 m_{1}
\end{array}\right)\left(\begin{array}{c}
m+1 \\
m_{1}
\end{array}\right)^{-3} \\
& \times \frac{3\left(m-m_{1}+1\right)}{3(m+1)} C_{0}^{2} A_{6}^{m+1}\left(\max \left(1, m_{1}\right)\right)^{-4 / 3} \\
& \times\left(\max \left(1, m+1-m_{1}\right)\right)^{-4 / 3}
\end{aligned}
$$

$$
\begin{aligned}
\leq & 3^{7} \sum_{m_{1}=0}^{m} \frac{\left(\max \left(1, m_{1}\right)\right)^{2 / 3}\left(m+1-m_{1}\right)^{2 / 3}}{(m+1)^{2 / 3}} \\
& \times C_{0}^{2} A_{6}^{m+1}\left(\max \left(1, m_{1}\right)\right)^{-4 / 3}\left(m+1-m_{1}\right)^{-4 / 3}
\end{aligned}
$$

$$
=3^{7} C_{0}^{2}(m+1)^{-2 / 3} A_{6}^{m+1} \sum_{m_{1}=0}^{m}\left(\max \left(1, m_{1}\right)\right)^{-2 / 3}\left(m+1-m_{1}\right)^{-2 / 3} \text {. }
$$

Using Schwarz' inequality, we have

$$
\begin{aligned}
& \sum_{m_{1}=0}^{m}\left(\max \left(1, m_{1}\right)\right)^{-2 / 3}\left(m+1-m_{1}\right)^{-2 / 3} \\
& \quad=(m+1)^{-2 / 3}+\sum_{m_{1}=1}^{m} m_{1}^{-2 / 3}\left(m+1-m_{1}\right)^{-2 / 3} \\
& \quad \leq(m+1)^{-2 / 3}+\left(\sum_{m_{1}=1}^{m} m_{1}^{-4 / 3}\right)^{1 / 2}\left(\sum_{m_{1}=1}^{m} m_{1}^{-4 / 3}\right)^{1 / 2} \leq C_{1} .
\end{aligned}
$$

Hence we have by (4.19)

$$
I_{2,2,1} \cdot \frac{A_{3}^{l+m+1}}{((l+m+1) !)^{\sigma^{\prime}}} \leq 3^{7} C_{0}^{2} C_{1} A_{6}^{m+1} .
$$

In the same way as in the proofs of (4.10)-(4.11) we have by (4.18) 
(4.21) $I_{2,2,2} \cdot \frac{A_{3}^{l+m+1}}{((l+m+1) !)^{\sigma^{\prime}}}$

$$
\begin{aligned}
\leq & 3 \sum_{m_{1}=0}^{m}\left(\begin{array}{c}
3 m \\
3 m_{1}
\end{array}\right)\left(\begin{array}{c}
m \\
m_{1}
\end{array}\right)^{-3} \\
& \times A^{2} C_{0}^{2} A_{6}^{m} A_{3} \frac{(3 m+2)(3 m+1)}{\left(3 m_{1}+1\right)\left(3 m-3 m_{1}+1\right)} \frac{1}{(m+1)^{3}} \\
\leq & 3^{9} A^{2} C_{0}^{2} A_{3} A_{6}^{m}
\end{aligned}
$$

(4.22) $I_{2,2,3} \cdot \frac{A_{3}^{l+m+1}}{((l+m+1) !)^{\sigma^{\prime}}}$

$\leq 3 \sum_{m_{1}=0}^{m}\left(\begin{array}{c}3 m \\ 3 m_{1}\end{array}\right)\left(\begin{array}{c}m \\ m_{1}\end{array}\right)^{-3} A^{2} C_{0}^{2} A_{6}^{m} A_{3} \frac{(3 m+2)(3 m+1)}{\left(3 m_{1}+2\right)\left(3 m_{1}+1\right)} \frac{1}{(m+1)^{3}}$

$\leq 3^{9} A^{2} C_{0}^{2} A_{3} A_{6}^{m}$,

Hence (4.20)-(4.22) imply that (4.6) holds true for $k=2$ if

$$
\frac{1}{4} A_{6}=2|\lambda| C 3^{9} A C_{0} A_{3} \quad \text { and } \quad 3^{7} C C_{0} C_{1}|\lambda|=\frac{A}{4} .
$$

From (4.9)-(4.11), (4.14)-(4.16), (4.20)-(4.22), and (4.12), (4.17), (4.23) it follows that (4.6) holds for

$$
A_{6}=2\left(1+2^{4} 3^{18}|\lambda|^{2} C^{2} C_{0}^{2} C_{1}\right) A_{3} .
$$

From (4.5) and (4.6) we have (4.2) and (4.3) under the condition

$$
A_{5}<\frac{A_{3}}{2\left(1+\left(1+2^{4} 3^{18}|\lambda|^{2} C^{2} C_{0}^{2} C_{1}\right) A_{3}\right)} .
$$

This completes the proof of Lemma 4.1.

Q.E.D.

Proof of Lemma 4.2. - For simplicity we denote $\|\cdot\|_{3}$ by $\|\cdot\|_{H^{3}(-R, R)}$. Since

$$
\frac{3^{3 m}}{(3 m+2) !} \leq \frac{1}{(m !)^{3}}
$$

Vol. 12, $n^{\circ} 6-1995$. 
we have

$$
\begin{aligned}
& \sum_{m=0}^{\infty} \frac{\left(|t|^{1 / 3} A_{4}\right)^{m}}{(m !)^{\sigma^{\prime} / 3}}\left\|\partial_{x}^{m} u\right\|_{3} \\
& =\sum_{m=0}^{\infty}\left(\frac{\left(|t|^{1 / 3} A_{4}\right)^{3 m}}{((3 m) !)^{\sigma^{\prime} / 3}}\left\|\partial_{x}^{3 m} u\right\|_{3}+\frac{\left(|t|^{1 / 3} A_{4}\right)^{3 m+1}}{((3 m+1) !)^{\sigma^{\prime} / 3}}\left\|\partial_{x}^{3 m+1} u\right\|_{3}\right. \\
& \left.+\frac{\left(|t|^{1 / 3} A_{4}\right)^{3 m+2}}{((3 m+2) !)^{\sigma^{\prime} / 3}}\left\|\partial_{x}^{3 m+2} u\right\|_{3}\right) \quad\left(\sigma^{\prime}=\max (\sigma, 3)\right) \\
& \leq \sum_{m=0}^{\infty}\left(((3 m+2)(3 m+1))^{\sigma^{\prime} / 3} \cdot\left(\frac{|t|^{1 / 3} A_{4}}{3}\right)^{3 m} \frac{1}{(m !)^{\sigma^{\prime}}}\left\|\partial_{x}^{3 m} u\right\|_{3}\right. \\
& +3(3 m+2)^{\sigma^{\prime} / 3} \cdot\left(\frac{|t|^{1 / 3} A_{4}}{3}\right)^{3 m+1} \frac{1}{(m !)^{\sigma^{\prime}}}\left\|\partial_{x}^{3 m+1} u\right\|_{3} \\
& \left.+3^{2} \cdot\left(\frac{|t|^{1 / 3} A_{4}}{3}\right)^{3 m+2} \frac{1}{(m !)^{\sigma^{\prime}}}\left\|\partial_{x}^{3 m+2} u\right\|_{3}\right) \\
& \leq \text { Const } \cdot \sum_{m=0}^{\infty}\left(\left(\frac{|t|^{1 / 3} A_{4}^{\prime}}{3}\right)^{3 m} \frac{1}{(m !)^{\sigma^{\prime}}}\left\|\partial_{x}^{3 m} u\right\|_{3}\right. \\
& +\left(\frac{|t|^{1 / 3} A_{4}^{\prime}}{3}\right)^{3 m+1} \frac{1}{(m !)^{\sigma^{\prime}}}\left\|\partial_{x}^{3 m+1} u\right\|_{3} \\
& \left.+\left(\frac{|t|^{1 / 3} A_{4}}{3}\right)^{3 m+2} \frac{1}{(m !)^{\sigma^{\prime}}}\left\|\partial_{x}^{3 m+2} u\right\|_{3}\right) \text { for } A_{4}^{\prime}>A_{4} \text {. } \\
& \leq \text { Const } \cdot \sum_{m=0}^{\infty}\left(\left(1+\frac{|t|^{1 / 3} A_{4}^{\prime}}{3}+\left(\frac{|t|^{1 / 3} A_{4}^{\prime}}{3}\right)^{2}\right) \frac{\left(|t| A_{5}^{\prime}\right)^{m}}{(m !)^{\sigma^{\prime}}}\left\|\partial_{x}^{3 m} u\right\|_{3}\right. \\
& \left.+\left(\frac{|t|^{1 / 3} A_{4}^{\prime}}{3}+\left(\frac{|t|^{1 / 3} A_{4}^{\prime}}{3}\right)^{2}\right) \frac{\left(|t| A_{5}^{\prime}\right)^{m}}{(m !)^{\sigma^{\prime}}}\left\|\partial_{x}^{3(m+1)} u\right\|_{3}\right) \text { for } A_{5}^{\prime}>\left(A_{4}^{\prime} / 3\right)^{3} \text {. }
\end{aligned}
$$

Therefore we have

$$
\begin{aligned}
& \sum_{m=0}^{\infty} \frac{\left(|t|^{1 / 3} A_{4}\right)^{m}}{(m !)^{\sigma^{\prime} / 3}}\left\|\partial_{x}^{m} u\right\|_{3} \\
& \quad \leq \text { Const } \cdot\left(1+|t|^{1 / 3}+|t|^{2 / 3}\right) \sum_{m=0}^{\infty} \frac{\left(|t| A_{5}\right)^{m}}{(m !)^{\sigma^{\prime}}}\left\|\partial_{x}^{3 m} u\right\|_{3}<\infty \\
& \quad \text { for } A_{5}>A_{5}^{\prime} .
\end{aligned}
$$


Hence, if we take $A_{4}$ such that

$$
A_{4}<3 A_{5}^{1 / 3}
$$

we have Lemma 4.2. We note here that by (4.25) and (4.26), when $p=2$, it is sufficient to take $A_{4}$ satisfying

$$
A_{4}<3\left(\frac{A_{3}}{2\left(1+\left(1+2^{4} \cdot 3^{18}|\lambda|^{2} C^{2} C_{0}^{2} C_{1}\right) A_{3}\right)}\right)^{1 / 3}
$$

to obtain the result.

Q.E.D.

\section{PROOFS OF THEOREMS 1.2-1.2' (NLS)}

The following two propositions were proved in [H-K.K, Propositions 3.3-3.4].

Proposition 5.1. - We assume that $\sigma \geq 1$ and

$$
\psi \in G_{\sigma}^{A_{1} A_{2}}\left(x \partial_{x}, \partial_{x} ; H^{2}\right) .
$$

Then there exist $A_{3}, T$ and a unique solution $u$ of (NLS) such that

$$
\begin{gathered}
u \in C\left([-T, T] ; H^{2}\right), \\
u \in G_{\sigma}^{|t| A_{3}}\left(\partial_{t} ; H^{2}(-R, R)\right) \quad \text { for } \quad t \in[-T, T],
\end{gathered}
$$

where

$$
A_{3}<\min \left\{\frac{2 A_{1}}{1+2 A_{1}}, \frac{2 A_{2}}{3 A_{2}+e^{1 / 2}(1+R)}\right\} .
$$

Proposition 5.2. - We assume that $\sigma \geq 1$ and

$$
\psi \in G_{\sigma}^{A_{1} A_{2}}\left(x \partial_{x}, \partial_{x} ; H^{2}\right) \cap G_{\sigma}^{A_{1} A_{2}}\left(x \partial_{x}, \partial_{x} ; L^{1}\right) .
$$

Furthermore we assume that

$$
\epsilon_{1}=\|\psi\|_{G_{\sigma}^{A_{1} A_{2}}\left(x \partial_{x}, \partial_{x} ; H^{2}\right)}+\|\psi\|_{G_{\sigma}^{A_{1} A_{2}}\left(x \partial_{x}, \partial_{x} ; L^{1}\right)}
$$

is sufficiently small and $G(u, \bar{u})$ satisfies the following growth condition

$$
|G(s, \bar{s})| \leq \text { Const. }|s|^{p} \text { for }|s|<1,
$$

Vol. $12, n^{\circ} 6-1995$. 
where $p$ is an integer satisfying $p \geq 5$. Then there exist $A_{3}$ and a unique solution $u$ of (NLS) such that

$$
\begin{gathered}
u \in C\left(\mathbb{R} ; H^{2}\right), \\
u \in G_{\sigma}^{|t| A_{3}}\left(\partial_{t} ; H^{2}(-R, R)\right) \text { for any } t \in \mathbb{R}, \\
\|u(t)\|_{G_{\sigma}^{A_{1} A_{2}}\left(x \partial_{x}, \partial_{x} ; L^{\infty}(-R, R)\right)} \leq \text { Const. } \epsilon_{1}(1+|t|)^{-1 / 2} \text { for any } t \in \mathbb{R},
\end{gathered}
$$

where

$$
A_{3}<\min \left\{\frac{2 A_{1}}{1+2 A_{1}}, \frac{2 A_{2}}{3 A_{2}+e^{1 / 2}(1+R)}\right\}
$$

We now prove Theorems 1.2 and 1.2'

Proofs of Theorems 1.2-1.2'. - From Proposition 5.1-5.2 it is sufficient to prove that there exists a positive constant $A_{4}$ such that

$$
u \in G_{\max (\sigma / 2,1)}^{|t|^{1 / 2} A_{4}}\left(\partial_{x} ; H^{2}(-R, R)\right)
$$

The proof is obtained in the same way as in the proof of Proposition 4.1, and so we only give the outline. We first prove there exists a positive constant $A_{5}$ such that

$$
u \in G_{\max (\sigma, 2)}^{|t| A_{5}}\left(\partial_{x}^{2} ; H^{2}(-R, R)\right)
$$

It suffices to show it for $t=1$. By Propositions 5.1-5.2 we have

$$
C_{0}=\sum_{l=0}^{\infty} \frac{A_{3}^{l}}{(l !)^{\sigma}}\left\|\partial_{t}^{l} u\right\|_{H^{2}(-R, R)}<\infty
$$

which implies

$$
\frac{A_{3}^{l}}{(l !)^{\sigma}}\left\|\partial_{t}^{l} u\right\|_{H^{2}(-R, R)} \leq C_{0}
$$

We prove by induction with respect to $m$ that there exists a positive constant $A_{6}$ such that

$$
\frac{A_{3}^{l+m}}{((l+m) !)^{\sigma^{\prime}}}\left\|\partial_{t}^{l} \partial_{x}^{2 m} \partial_{x}^{k} u\right\|_{H^{1}(-R, R)} \leq C_{0} A_{6}^{m}, \quad \text { for } \quad k=0,1
$$


for $l, m \in \mathbb{N} \cup\{0\}, \sigma^{\prime}=\max (\sigma, 2)$. If (5.4) is valid, then taking $l=0$, we get (5.2) with

$$
A_{5}=\frac{A_{3}}{A_{6}}-\epsilon \quad(\epsilon>0)
$$

In what follows for simplicity we let

$$
\|\cdot\|_{H^{1}(-R, R)}=\|\cdot\|_{1} \text { and } M=\partial_{x}^{2} .
$$

It is clear that (5.4) holds true for all $l$ when $m=0$ by (5.3). We assume that (5.4) is valid for all $l, m$ and $k=0,1$. We only consider the case

$$
i \partial_{t} u+\frac{1}{2} \Delta u=u^{2}
$$

since the general nonlinearity can be treated by induction. We have by an elementary calculation and Sobolev's inequality

$$
I_{2,0} \equiv\left\|\partial_{t}^{l} \partial_{x}^{2 m}\left(u^{2}\right)\right\|_{1}
$$

$\leq \sum_{l^{\prime}=0}^{l} \sum_{m^{\prime}}^{2 m}\left(\begin{array}{c}l \\ l^{\prime}\end{array}\right)\left(\begin{array}{c}2 m \\ m^{\prime}\end{array}\right)\left\|\partial_{t}^{l^{\prime}} \partial_{x}^{m^{\prime}} u \cdot \partial_{t}^{l-l^{\prime}} \partial_{x}^{2 m-m^{\prime}} u\right\|_{1}$

$=\sum_{l^{\prime}=0}^{l} \sum_{m_{1}=0}^{m}\left(\begin{array}{c}l \\ l^{\prime}\end{array}\right)\left(\begin{array}{c}2 m \\ 2 m_{1}\end{array}\right)\left\|\partial_{t}^{l^{\prime}} M^{m_{1}} u \cdot \partial_{t}^{l-l^{\prime}} M^{m-m_{1}} u\right\|_{1}$

$+\sum_{l^{\prime}=0}^{l} \sum_{m_{1}=0}^{m-1}\left(\begin{array}{c}l \\ l^{\prime}\end{array}\right)\left(\begin{array}{c}2 m \\ 2 m_{1}+1\end{array}\right)\left\|\partial_{t}^{l^{\prime}} M^{m_{1}} \partial_{x} u \cdot \partial_{t}^{l-l^{\prime}} M^{m-m_{1}-1} \partial_{x} u\right\|_{1}$

$$
\begin{aligned}
\leq & C \sum_{l^{\prime}=0}^{l} \sum_{m_{1}=0}^{m}\left(\begin{array}{c}
l \\
l^{\prime}
\end{array}\right)\left(\begin{array}{c}
2 m \\
2 m_{1}
\end{array}\right)\left\|\partial_{t}^{l^{\prime}} M^{m_{1}} u\right\|_{1}\left\|\partial_{t}^{l-l^{\prime}} M^{m-m_{1}} u\right\|_{1} \\
& +C \sum_{l^{\prime}=0}^{l} \sum_{m_{1}=0}^{m-1}\left(\begin{array}{c}
l \\
l^{\prime}
\end{array}\right)\left(\begin{array}{c}
2 m \\
2 m_{1}+1
\end{array}\right)\left\|\partial_{t}^{l^{\prime}} M^{m_{1}} \partial_{x} u\right\|_{1}\left\|\partial_{t}^{l-l^{\prime}} M^{m-m_{1}-1} \partial_{x} u\right\|_{1}
\end{aligned}
$$

$\equiv C\left(I_{2,0,1}+I_{2,0,2}\right)$

Vol. $12, n^{\circ}$ 6-1995. 
In the same way as in the proof of (4.9)

$$
\begin{aligned}
& I_{2,0,1} \frac{A_{3}^{l+m+1}}{((l+m+1) !)^{\sigma^{\prime}}} \\
& \quad \leq 3 \sum_{m_{1}=0}^{m}\left(\begin{array}{c}
2 m \\
2 m_{1}
\end{array}\right)\left(\begin{array}{c}
m \\
m_{1}
\end{array}\right)^{-2} \frac{C_{0}^{2} A_{3} A_{6}^{m}}{(m+1)^{2}} \\
& \quad \leq 3 \cdot 2^{6} \sum_{m_{1}=0}^{m} \frac{\left(\max \left(1, m_{1}\right)\right)\left(\max \left(1, m-m_{1}\right)\right)}{m} \cdot \frac{C_{0}^{2} A_{3} A_{6}^{m}}{(m+1)^{2}}
\end{aligned}
$$

(by Lemma 2.10)

$$
\leq 3 \cdot 2^{6} C_{0}^{2} A_{3} A_{6}^{m}
$$

and in the same way as in the proof of (4.11) we have

$$
\begin{aligned}
& I_{2,0,2} \frac{A_{3}^{l+m+1}}{((l+m+1) !)^{\sigma^{\prime}}} \\
& \quad \leq 3 \sum_{m_{1}=0}^{m-1}\left(\begin{array}{c}
2 m \\
2 m_{1}+1
\end{array}\right)\left(\begin{array}{c}
m \\
m_{1}
\end{array}\right)^{-2} \frac{C_{0}^{2} A_{3}^{2} A_{6}^{m-1}}{(m+1)^{2}\left(m-m_{1}\right)^{2}} \\
& \quad=3 \sum_{m_{1}=0}^{m-1}\left(\begin{array}{c}
2 m \\
2 m_{1}
\end{array}\right)\left(\begin{array}{c}
m \\
m_{1}
\end{array}\right)^{-2} \frac{2\left(m-m_{1}\right)}{2 m_{1}+1} \frac{C_{0}^{2} A_{3}^{2} A_{6}^{m-1}}{(m+1)^{2}\left(m-m_{1}\right)^{2}} \\
& \leq 3 \cdot 2^{7} C_{0}^{2} A_{3} A_{6}^{m} \quad \text { (by Lemma 2.10). }
\end{aligned}
$$

We also have (see (4.13))

$$
\begin{aligned}
& \frac{1}{C} I_{2,1} \equiv \frac{1}{C}\left\|\partial_{t}^{l} \partial_{x}^{2 m} \partial_{x}\left(u^{2}\right)\right\|_{1} \\
& \leq \sum_{l^{\prime}=0}^{l} \sum_{m_{1}=0}^{m}\left(\begin{array}{c}
l \\
l^{\prime}
\end{array}\right)\left(\begin{array}{c}
2 m+1 \\
2 m_{1}
\end{array}\right)\left\|\partial_{t}^{l^{\prime}} M^{m_{1}} u\right\|_{1}\left\|\partial_{t}^{l-l^{\prime}} M^{m-m_{1}} \partial_{x} u\right\|_{1} \\
& \quad+\sum_{l^{\prime}=0}^{l} \sum_{m_{1}=0}^{m-1}\left(\begin{array}{l}
l \\
l^{\prime}
\end{array}\right)\left(\begin{array}{c}
2 m+1 \\
2 m_{1}+1
\end{array}\right)\left\|\partial_{t}^{l^{\prime}} M^{m_{1}} \partial_{x} u\right\|_{1}\left\|\partial_{t}^{l-l^{\prime}} M^{m-m_{1}-1} \partial_{x} u\right\|_{1} \\
& \equiv I_{2,1,1}+I_{2,1,2} .
\end{aligned}
$$

In the same way as we proved (4.14) and (4.15) we obtain by Lemma 2.10

$$
\left(I_{2,1,1}+I_{2,1,2}\right) \frac{A_{3}^{l+m+1}}{((l+m+1) !)^{\sigma^{\prime}}} \leq 3 \cdot 2^{8} C_{0}^{2} A_{3} A_{6}^{m} .
$$


From (5.6)-(5.8) we see that if we take $A_{6}$ such that

$$
A_{6}=3 \cdot 2^{1} 0 C_{0} C A_{3}
$$

then

$$
\left\|\partial_{t}^{l} M^{m} \partial_{x}^{k}\left(u^{2}\right)\right\|_{1} \leq \frac{C_{0}}{2} A_{6}^{m+1} .
$$

On the other hand, the solution of (NLS2) satisfies

$$
\left\|\partial_{t}^{l} M^{m+1} \partial_{x}^{k} u\right\|_{1} \leq\left\|\partial_{t}^{l+1} M^{m} \partial_{x}^{k} u\right\|_{1}+\left\|\partial_{t}^{l} M^{m} \partial_{x}^{k}\left(u^{2}\right)\right\|_{1} .
$$

By the induction assumption, we see that the first term of the right hand side is estimated from above by

$$
C_{0} A_{6}^{m}=\frac{1}{A_{6}} C_{0} A_{6}^{m+1} \leq \frac{C_{0}}{2} A_{6}^{m+1} \quad \text { if } \quad A_{6} \geq 2 .
$$

Hence, we have (5.4) by (5.9) and (5.10) if

$$
A_{6}=2+3 \cdot 2^{1} 0 C_{0} C A_{3} .
$$

Using (5.4) and the same argument as in Lemma 4.2, we obtain the desired estimate (5.1), provided that $A_{4}$ satisfies

$$
A_{4}<2\left(\frac{A_{3}}{2\left(1+3 \cdot 2^{9} C C_{0} A_{3}\right)}\right)^{1 / 2} .
$$

Q.E.D.

\section{ACKNOWLEDGEMENTS}

This work was started when the second and third authors stayed at the University of Paris-Sud. The authors (N. H and K. K) would like to express their hearty thanks to Professors T. Cazenave, J. Ginibre, G. Lebeau and J. C. Saut for their kind hospitality at Université de Paris-Sud and Université Pierre-et-Marie-Curie. The second author was partially supported by the Gunma University Foundation for Science and Technology. 


\section{REFERENCES}

[A]

[B]

[Co-Sau]

[Cr-Kap-St]

[G-Vel]

$[\mathrm{H}]$

$[\mathrm{H}-\mathrm{N}-\mathrm{T}]$

[H-K.K]

$[\mathrm{H}-\mathrm{O}]$

[H-Sai]

[Ka]

$[\mathrm{Ka}-\mathbf{M}]$

[Ke-P-V 1]

[Ke-P-V 2]

[Ke-P-V 3]

[Ke-P-V 4]

$[$ Ke-P-V 5$]$

$[\mathrm{Kr}-\mathrm{F}]$

H. AIKAWA, Infinite order Sobolev spaces, analytic continuation and polynomial expansions, Complex Variables, Vol. 18, 1992, pp. 253-266.

A. DE BOuARD, Analytic solutions to nonelliptic nonlinear Schrödinger equations, J. Diff. Eqs., Vol. 104, 1993, pp. 196-213.

P. Constantin and J. C. Saut, Local smoothing properties of dispersive equations, J. Amer. Math. Soc., Vol. 1, 1988, pp. 413-439.

W. Craig, K. Kappeler and W. A. Strauss, Gain of regularity for solutions of KdV type, Ann. Inst. Henri Poincaré, Analyse non linéaire, Vol. 9, 1992, pp. 147-186.

J. GiNIBRE and G. VeLo, On a class of nonlinear Schrödinger equations I, II, J. Funct. Anal., Vol. 32, 1979, pp. 1-32, 33-71.

N. HAYASHI, Analyticity of solutions of the Korteweg-de Vries equation, SIAM J. Math. Anal., Vol. 22, 1991, pp. 1738-1745.

N. HAYASH, K. NAKAmitsu and M. Tsutsumi, On solutions of the initial value problem for the nonlinear Schrödinger equation in one space dimension, Math. Z., Vol. 192, 1986, pp. 637-650.

N. HAYASHI and K. KATO, Regularity of solutions in time to nonlinear Schrödinger equations, J. Funct. Anal., 1994 (to appear).

N. HAYASH and T. OZAWA, Remarks on nonlinear Schrödinger equations in one space dimensions, Diff. and Integral Eqs., Vol. 7, 1994, pp. 453-461.

N. HAYASHI and S. SAITOH, Analyticity and global existence of small solutions to some nonlinear Schrödinger equations, Commun. Math. Phys., Vol. 129, 1990 , pp. 27-42.

T. KATO, On the Cauchy problem for the (generalized) Korteweg-de Vries equation, Stud. Appl. Math. Adv. in Math. Supplementary Studies, Vol. 18, 1983, pp. 93-128.

T. KATO and K. MASUDA, Nonlinear evolution equations and analyticity I, Ann. Inst. Henri Poincaré Analyse non linéaire, Vol. 3, 1986, pp. 455-467.

C. E. KeniG, G. PonCE and L. VeGAA, Oscillatory integrals and regularity of dipersive equations, Indiana Univ. Math. J., Vol. 40, 1991, pp. 33-69.

C. E. KenIG, G. Ponce and L. VegaA, Small solutions to nonlinear Schrödinger equations, Ann. Inst. Henri Poincaré, Analyse non linéaire, Vol. 10, 1993, pp. 255-288.

C. E. KenIG, G. PONCE and L. VeGAA, Higher order non-linear dispersive equations, preprint, 1992.

C. E. KeNIG, G. PONCE and L. VEGAA, On the hierarchy of the generalized KdV equations, Proc. Lyon Workshop on Singular Limits of Dispersive Waves, 1993 (to appear).

C. E. KenIG, G. Ponce and L. VegaA, Well-posedness and scattering results for the generalized Korteweg-de Vries equations via the contraction principle, Comm. Pure, Appl. Math., Vol. 46, 1993, pp. 527-620.

H. KomaTsU, "Introduction to distributions, ultradistributions and hyperfunctions (in Japanese)", Iwanami Kisosugaku, Iwanami shoten, Tokyo, 1978.

S. N. KruzhKov and A. V. Faminskil, Generalized solutions to the Cauchy problem for the Korteweg-de Vries equation, Math. U.S.S.R. Sbornik, Vol. 48, 1984, pp. 93-138.

C. LAUREY, The Cauchy problem for a third order nolinear Schrödinger equations, preprint, 1993.

G. PONCE, Regularity of solutions to nonlinear dispersive equations, J. Diff. Eqs., Vol. 78, 1989, pp. 122-135. 
[Sj] P. SJöLIN, Regularity of solutions to the Schrödinger equations, Duke Math. J., Vol. 55, 1987, pp. 699-715.

[St] W. STRaUSs, Dispersion of low energy waves for two conservative equations, Arch. Rational Mech. Anal., Vol. 55, 1974, pp. 86-92.

[V] L. VEGA, Schrödinger equations : pointwise convergence to the initial data, Proc. Amer. Marh. Soc., Vol. 102, pp. 874-878.

(Manuscript received February 17, 1994.) 